\title{
ACCURATE ATMOSPHERIC PARAMETERS AT MODERATE RESOLUTION USING SPECTRAL INDICES: PRELIMINARY APPLICATION TO THE MARVELS SURVEY*
}

\author{
Luan Ghezzi $^{1,2}$, Letícia Dutra-Ferreira $^{2,3}$, Diego Lorenzo-Oliveira $^{2,3}$, Gustavo F. Porto De Mello ${ }^{2,3}$, \\ Basílio X. Santiago ${ }^{2,4}$, Nathan De LeE ${ }^{5,6,7}$, Brian L. LeE ${ }^{7,8}$, Luiz N. Da Costa ${ }^{1,2}$, Marcio A. G. Maia ${ }^{1,2}$, \\ Ricardo L. C. OGando ${ }^{1,2}$, John P. Wisniewski ${ }^{9}$, Jonay I. González Hernández ${ }^{10,11}$, Keivan G. Stassun ${ }^{6,12}$, \\ Scott W. Fleming ${ }^{13,14}$, Donald P. Schneider ${ }^{15,16}$, Suvrath Mahadevan ${ }^{15,16}$, Phillip Cargile ${ }^{6}$, Jian Ge $^{7}$, \\ Joshua PePper ${ }^{6,17}$, Ji Wang ${ }^{18}$, And Martin PAegerT ${ }^{6}$ \\ ${ }^{1}$ Observatório Nacional, Rua Gal. José Cristino 77, Rio de Janeiro, RJ 20921-400, Brazil \\ ${ }^{2}$ Laboratório Interinstitucional de e-Astronomia - LIneA, Rua Gal. José Cristino 77, Rio de Janeiro, RJ 20921-400, Brazil; luan@ @inea.gov.br \\ ${ }^{3}$ Universidade Federal do Rio de Janeiro, Observatório do Valongo, Ladeira do Pedro Antônio 43, Rio de Janeiro, RJ 20080-090, Brazil \\ ${ }^{4}$ Instituto de Física, UFRGS, Caixa Postal 15051, Porto Alegre, RS 91501-970, Brazil \\ ${ }^{5}$ Department of Physics and Geology, Northern Kentucky University, Highland Heights, KY 41099, USA \\ ${ }^{6}$ Department of Physics and Astronomy, Vanderbilt University, Nashville, TN 37235, USA \\ ${ }^{7}$ Department of Astronomy, University of Florida, 211 Bryant Space Science Center, Gainesville, FL 32611-2055, USA \\ ${ }^{8}$ Astronomy Department, University of Washington, Box 351580, Seattle, WA 98195, USA \\ ${ }^{9}$ H. L. Dodge Department of Physics and Astronomy, University of Oklahoma, 440 West Brooks St Norman, OK 73019, USA \\ ${ }^{10}$ Instituto de Astrofísica de Canarias (IAC), E-38205 La Laguna, Tenerife, Spain \\ ${ }^{11}$ Departamento de Astrofísica, Universidad de La Laguna, E-38206 La Laguna, Tenerife, Spain \\ ${ }^{12}$ Department of Physics, Fisk University, Nashville, TN, USA \\ ${ }^{13}$ Space Telescope Science Institute - STScI, 3700 San Martin Drive, Baltimore, MD 21218, USA \\ ${ }^{14}$ Computer Sciences Corporation, 3700 San Martin Drive, Baltimore, MD, USA \\ 15 Department of Astronomy and Astrophysics, The Pennsylvania State University, University Park, PA 16802, USA \\ ${ }^{16}$ Center for Exoplanets and Habitable Worlds, The Pennsylvania State University, University Park, PA 16802, USA \\ ${ }^{17}$ Department of Physics, Lehigh University, 16 Memorial Drive East, Bethlehem, PA 18015, USA \\ ${ }^{18}$ Department of Astronomy, Yale University, New Haven, CT 06511, USA \\ Received 2013 December 11; accepted 2014 July 1; published 2014 October 23
}

\begin{abstract}
Studies of Galactic chemical, and dynamical evolution in the solar neighborhood depend on the availability of precise atmospheric parameters (effective temperature $T_{\text {eff }}$, metallicity $[\mathrm{Fe} / \mathrm{H}]$, and surface gravity $\log g$ ) for solar-type stars. Many large-scale spectroscopic surveys operate at low to moderate spectral resolution for efficiency in observing large samples, which makes the stellar characterization difficult due to the high degree of blending of spectral features. Therefore, most surveys employ spectral synthesis, which is a powerful technique, but relies heavily on the completeness and accuracy of atomic line databases and can yield possibly correlated atmospheric parameters. In this work, we use an alternative method based on spectral indices to determine the atmospheric parameters of a sample of nearby FGK dwarfs and subgiants observed by the MARVELS survey at moderate resolving power $(R \sim 12,000)$. To avoid a time-consuming manual analysis, we have developed three codes to automatically normalize the observed spectra, measure the equivalent widths of the indices, and, through a comparison of those with values calculated with predetermined calibrations, estimate the atmospheric parameters of the stars. The calibrations were derived using a sample of 309 stars with precise stellar parameters obtained from the analysis of high-resolution FEROS spectra, permitting the low-resolution equivalent widths to be directly related to the stellar parameters. A validation test of the method was conducted with a sample of 30 MARVELS targets that also have reliable atmospheric parameters derived from the high-resolution spectra and spectroscopic analysis based on the excitation and ionization equilibria method. Our approach was able to recover the parameters within $80 \mathrm{~K}$ for $T_{\text {eff }}, 0.05 \mathrm{dex}$ for $[\mathrm{Fe} / \mathrm{H}]$, and $0.15 \mathrm{dex}$ for $\log g$, values that are lower than or equal to the typical external uncertainties found between different high-resolution analyses. An additional test was performed with a subsample of 138 stars from the ELODIE stellar library, and the literature atmospheric parameters were recovered within $125 \mathrm{~K}$ for $T_{\text {eff }}, 0.10 \mathrm{dex}$ for $[\mathrm{Fe} / \mathrm{H}]$, and $0.29 \mathrm{dex}$ for $\log g$. These precisions are consistent with or better than those provided by the pipelines of surveys operating with similar resolutions. These results show that the spectral indices are a competitive tool to characterize stars with intermediate resolution spectra.
\end{abstract}

Key words: solar neighborhood - stars: atmospheres - stars: fundamental parameters - stars: solar-type techniques: spectroscopic

Online-only material: color figures, supplemental data

\section{INTRODUCTION}

Solar-type stars are prime targets for many studies in astrophysics. Their spectra are rich in metallic lines, which allow precise determinations of the fundamental stellar parameters

\footnotetext{
* Based on observations obtained with the $2.2 \mathrm{~m} \mathrm{MPG}$ telescope at the European Southern Observatory (La Silla, Chile), under the agreement ESO-Observatório Nacional/MCT, and the Sloan Digital Sky Survey, which is owned and operated by the Astrophysical Research Consortium.
}

and elemental abundances through different well-established techniques (e.g., excitation and ionization equilibria or spectral synthesis). They are also long-lived and have a large age dispersion, probing a considerable fraction of the history of the Milky Way. Furthermore, the compositions of their atmospheres remain almost unchanged (with the exception of $\mathrm{Li}, \mathrm{Be}$, and B) throughout their evolution on the main sequence. All these properties make solar-type stars the ideal candidates to study time-dependent processes, such as the chemical evolution 
in the solar neighborhood, and many successful examples of this application can be found in the literature (e.g., Edvardsson et al. 1993; Casagrande et al. 2011).

The addition of radial or spatial velocities for these objects provides an approach to study dynamical processes within the disk, such as radial migration and kinematical heating (e.g., Haywood 2008). Local stellar samples with both kinematical and chemical information may also be used to identify stars from different Galactic components within the thin-disk dominated population in the solar neighborhood (Gratton et al. 2003; Bensby \& Feltzing 2006; Karatas \& Klement 2012). In order to improve the accuracy of these studies and to extend them over larger volumes, massive spectroscopic surveys such as SEGUE (Yanny et al. 2009) and RAVE (Steinmetz et al. 2006) have been developed, with additional projects expected in the near future (e.g., GALAH; Zucker et al. 2012).

Although its main scientific goal is the study of the formation and evolution of giant planets, brown dwarfs, and low-mass stars, it was soon realized that the Multi-object APO Radial Velocity Exoplanet Large-area Survey (MARVELS; Ge et al. 2008, 2009; Ge \& Eisenstein 2009) could also contribute to a better understanding of the chemical and kinematical evolution of the solar neighborhood. During its four-year operation (2008-2012) as part of the third phase of the Sloan Digital Sky Survey (SDSS-III; York et al. 2000; Eisenstein et al. 2011), a sample of $\sim 3300$ FGK stars with $7.6 \leqslant V \leqslant 12.0$ (many of which were never previously analyzed) had their radial velocities (RVs) monitored in the search for companions. The targets were selected according to a limited number of welldefined criteria that did not explicitly include any cuts based on the metallicities, activity levels, and ages of the stars (for more details, see Lee et al. 2011). The final sample thus presents a well-characterized selection function, providing a statistically homogeneous data set.

The kinematical analysis will benefit from the precise RVs delivered by the MARVELS survey $\left(\sigma_{\mathrm{RV}} \lesssim 100 \mathrm{~m} \mathrm{~s}^{-1}\right)$. The chemical analysis, on the other hand, depends on the availability of precise atmospheric parameters (effective temperature, $T_{\text {eff }}$, and surface gravity, $\log g$ ) and chemical abundances (metallicity, $[\mathrm{Fe} / \mathrm{H}]$, and $\alpha$-element content, $[\alpha / \mathrm{Fe}])$. Stars with RV variations suggestive of the presence of companions were selected for a more detailed study, which included the acquisition of high-resolution $(R \gtrsim 30,000)$ spectra to determine their fundamental parameters by applying standard spectroscopic techniques (excitation and ionization equilibria of $\mathrm{Fe}$ I and $\mathrm{Fe}$ II lines; see Wisniewski et al. 2012 for details).

Stars without detected companions, which correspond to $\sim 80 \%$ of the survey targets, were not subjected to a similar highresolution spectroscopic follow-up, and thus the stellar characterization had to rely solely on the MARVELS data. Although the numbers of visits for each target (typically $\gtrsim 20$ ) were able to produce final combined spectra with high signal-to-noise ratios ( $\mathrm{S} / \mathrm{N} \gtrsim 100)$, the moderate resolution $(R \sim 12,000)$ and somewhat limited wavelength range $(\sim 5000-5700 \AA)$ of the data prevented the usage of the classical analysis mentioned above. In this resolution regime, most of the lines are blended with neighboring features and the stellar characterization through the measurement of equivalent widths (EWs) of individual iron lines is not feasible.

Recent large spectroscopic surveys operating in the low-tointermediate resolution ( $\sim 2000-20,000)$ regime have developed pipelines that rely exclusively, or at least partially, on the spectral synthesis technique in which the stellar parameters are de- termined through a comparison of the observed spectra with an extensive library of previously calculated synthetic ones. As examples, we can mention the pipelines from SEGUE (SSPP; Lee et al. 2008; Smolinski et al. 2011), RAVE (Siebert et al. 2011; Kordopatis et al. 2013), LAMOST (Wu et al. 2011), and AMBRE (de Laverny et al. 2012; Worley et al. 2012). Although spectral fitting is a powerful technique and provides precise results for high-quality data (e.g., Valenti \& Fischer 2005), it has some drawbacks: (1) dependency on a very detailed and extensive list of spectral lines (many of which may have poorly determined atomic parameters), (2) the need to accurately know the broadening parameters (instrumental profile, macroturbulence, and rotational velocities), and (3) stronger correlations (relative to the excitation and ionization equilibria method) between the resulting atmospheric parameters (see, e.g., Torres et al. 2012). For the specific case of MARVELS, the relatively small wavelength coverage $(\Delta \lambda \simeq 700 \AA)$ combined with the moderate resolution can significantly decrease the accuracy of the spectral synthesis technique. Therefore, a different method is necessary to efficiently and accurately derive the parameters of the stars that did not have high-resolution follow-up spectra.

The purpose of this work is to develop and validate an alternative approach based on spectral indices-specific spectral regions combining multiple absorption lines into broad, blended features - to determine atmospheric parameters directly from the MARVELS spectra, without any other priors. Indices have been successfully applied before to derive information on mean stellar ages and metallicities of populations of galaxies and stars (e.g., Worthey et al. 1994; Trager et al. 2000; Sánchez-Blázquez et al. 2007; Ogando et al. 2008), as well as atmospheric parameters for target selection purposes in planet search surveys (e.g., Robinson et al. 2006, 2007). Because a manual object-by-object analysis of a numerous sample such as that from MARVELS is prohibitively time-consuming, we have developed three codes to automate the determination of stellar parameters.

This paper describes the proposed automatic approach to perform the stellar characterization of MARVELS targets and validates its results using data from the survey and the ELODIE stellar library (Prugniel \& Soubiran 2001, Prugniel et al. 2007; see Web site ${ }^{19}$ for the most updated 3.1 version). The paper is organized as follows. Section 2 presents the data used to build the calibrations that were later adopted for the derivation of the atmospheric parameters. A sample of MARVELS stars utilized to test the precision of the results is also described. The definition of the spectral indices is detailed in Section 3, while Section 4 is devoted to a thorough description of the method. The four steps of our analysis (normalization of the spectra, measurements of EWs, construction of the calibrations, and derivation of atmospheric parameters) are explained in separate subsections. The results obtained with the spectral indices are shown in Section 5, along with a discussion regarding their accuracy. Finally, our concluding remarks are presented in Section 6.

\section{DATA}

Two different samples of stars were used in this work. The first is formed by well-characterized stars (through the analysis of high-resolution spectra) and was utilized to construct the calibrations used to obtain the atmospheric parameters $\left(T_{\text {eff }}\right.$, $[\mathrm{Fe} / \mathrm{H}]$, and $\log g$ ), thus being referred to as the calibration sample. The second contains a subset of MARVELS targets

\footnotetext{
19 http://www.obs.u-bordeaux1.fr/m2a/soubiran/elodie_library.html
} 

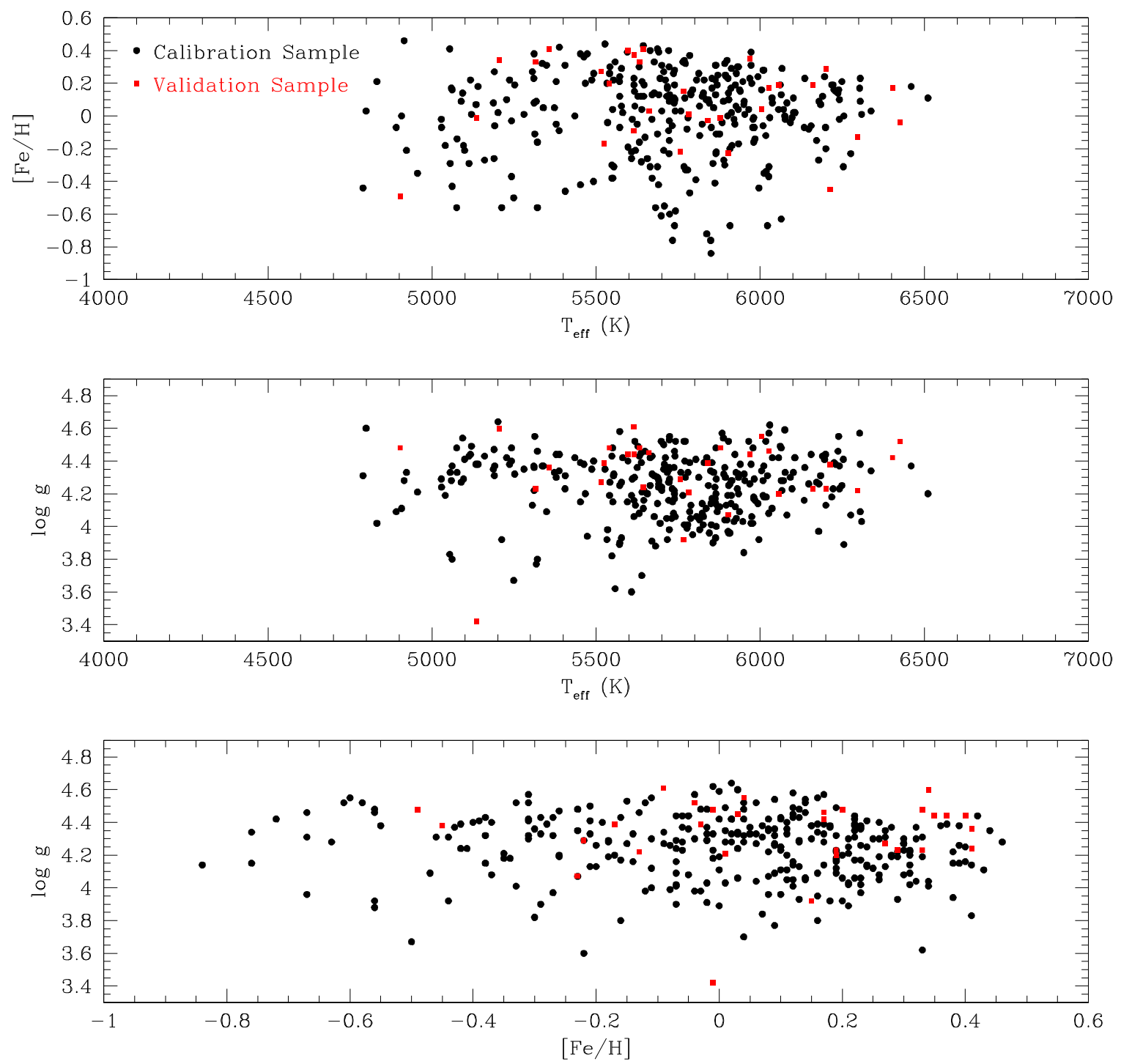

Figure 1. Atmospheric parameters $\left(T_{\text {eff }},[\mathrm{Fe} / \mathrm{H}]\right.$, and $\left.\log g\right)$ for the 309 stars in the calibration sample (black circles) and the 30 stars in the validation sample (red squares). The intervals covered by the calibration sample are: $4800 \mathrm{~K} \lesssim T_{\text {eff }} \lesssim 6500 \mathrm{~K}, 3.60 \lesssim \log g \lesssim 4.70$, and $-0.90 \lesssim[\mathrm{Fe} / \mathrm{H}] \lesssim+0.50$. The calibration stars also cover the parameter space of the MARVELS sample, represented here by the validation sample.

(A color version of this figure is available in the online journal.)

for which independent high-resolution spectra and precise atmospheric parameters are available. Because its purpose was to check the performance of the spectral indices approach, we called it the validation sample. Both samples are described in more detail below.

\subsection{Calibration Sample}

The calibration sample is composed of 309 stars; their highresolution spectra, effective temperatures, surface gravities, and metallicities were taken from Ghezzi et al. (2010a, 2010b) and del Peloso et al. (2005a, 2005b). Figure 1 shows the distribution of these stars (open circles) in parameter space; it is clear that this sample has a good coverage in the following intervals: $4800 \mathrm{~K} \lesssim T_{\text {eff }} \lesssim 6500 \mathrm{~K}, 3.60 \lesssim \log g \lesssim 4.70$, and $-0.90 \lesssim$ $[\mathrm{Fe} / \mathrm{H}] \lesssim+0.50$.

The majority of the stars were taken from Ghezzi et al. (2010a, 2010b), but the 17 stars classified as giants by the authors were not included here. This cut was done because their spectra are considerably different from those of dwarfs and subgiants and a proper analysis would require a distinct set of spectral indices.
The remaining 291 stars (262 dwarfs and 29 subgiants) all have high-resolution $(R \sim 48,000)$ and high-quality $(\mathrm{S} / \mathrm{N} \gtrsim 200$ per resolution element at $\lambda \sim 6700 \AA$ ) spectra obtained with the Fiber-fed Extended Range Optical Spectrograph (FEROS) spectrograph (Kaufer et al. 1999) attached to the MPG/ESO $2.2 \mathrm{~m}$ telescope (La Silla, Chile), under the agreement between ESO and Observatório Nacional (MCT). Additional details about the sample selection, observations, and data reduction are given in Ghezzi et al. (2010b). The authors also describe the homogeneous derivation of the atmospheric parameters $\left(T_{\text {eff }}\right.$ and $\left.\log g\right)$ and metallicities $([\mathrm{Fe} / \mathrm{H}])$ that were utilized in this work. In summary, the standard spectroscopic analysis based on the excitation and ionization equilibria of a carefully selected list of Fe I and Fe II lines was used to determine the results in an automated procedure.

In order to better populate the region $[\mathrm{Fe} / \mathrm{H}]<-0.50$ of the parameter space, the above sample was complemented with stars from del Peloso et al. (2005a, 2005b). Nine stars are common with Ghezzi et al. (2010a, 2010b) and were considered only for comparative purposes (see below). 
Table 1

Atmospheric Parameters for the MARVELS Candidates

\begin{tabular}{lcrcc}
\hline \hline Star & \multicolumn{1}{c}{$T_{\text {eff }}$} & \multicolumn{1}{c}{$[\mathrm{Fe} / \mathrm{H}]$} & $\log g$ & Reference \\
& $(\mathrm{K})$ & & & \\
\hline MC1 & $6297 \pm 28$ & $-0.13 \pm 0.04$ & $4.22 \pm 0.09$ & Wright et al. (2013) \\
MC2 & $5598 \pm 63$ & $0.40 \pm 0.09$ & $4.44 \pm 0.17$ & De Lee et al. (2013) \\
MC5 & $6214 \pm 38$ & $-0.45 \pm 0.06$ & $4.38 \pm 0.15$ & This work \\
MC6 & $6427 \pm 33$ & $-0.04 \pm 0.05$ & $4.52 \pm 0.14$ & Fleming et al. (2012) \\
MC7 & $5879 \pm 29$ & $-0.01 \pm 0.05$ & $4.48 \pm 0.15$ & Wisniewski et al. (2012) \\
MC11 & $6004 \pm 29$ & $0.04 \pm 0.05$ & $4.55 \pm 0.15$ & Jiang et al. (2013) \\
MC12 & $5903 \pm 42$ & $-0.23 \pm 0.07$ & $4.07 \pm 0.16$ & Ma et al. (2013) \\
MCKGS1-50 & $5540 \pm 39$ & $0.20 \pm 0.07$ & $4.48 \pm 0.23$ & This work \\
MCKGS1-52 & $6403 \pm 44$ & $0.17 \pm 0.07$ & $4.42 \pm 0.24$ & This work \\
MCKGS1-61 & $5757 \pm 43$ & $-0.22 \pm 0.07$ & $4.29 \pm 0.22$ & This work \\
MCKGS1-70 & $5135 \pm 39$ & $-0.01 \pm 0.08$ & $3.42 \pm 0.18$ & This work \\
MCKGS1-94 & $4903 \pm 47$ & $-0.49 \pm 0.08$ & $4.48 \pm 0.28$ & This work \\
MCKGS1-112 & $5782 \pm 42$ & $0.01 \pm 0.07$ & $4.21 \pm 0.23$ & This work \\
MCKGS1-135 & $5525 \pm 71$ & $-0.17 \pm 0.08$ & $4.39 \pm 0.23$ & This work \\
MCKGS1-153 & $5614 \pm 29$ & $-0.09 \pm 0.06$ & $4.61 \pm 0.09$ & This work \\
MCUF1-11 & $5315 \pm 44$ & $0.33 \pm 0.06$ & $4.23 \pm 0.19$ & This work \\
& & & & \\
& & & & \\
& & & &
\end{tabular}

The remaining 18 stars have high-resolution $(R \sim 48,000)$ and high-quality $(\mathrm{S} / \mathrm{N} \gtrsim 300)$ spectra acquired with the FEROS spectrograph fed by the ESO $1.52 \mathrm{~m}$ telescope. The details about the sample selection, observations, and data reduction for these objects can be seen in del Peloso et al. (2005a). A description of the iterative method used to determine the atmospheric parameters ( $T_{\text {eff }}$ and $\log g$ ) and metallicities $([\mathrm{Fe} / \mathrm{H}])$ adopted here are also provided by the authors. Briefly, the effective temperatures were estimated from the arithmetic mean of the values derived from photometric calibrations and $\mathrm{H} \alpha$ profile fitting. The surface gravities were derived using these effective temperatures and also stellar luminosities and masses. The luminosities were calculated from Hipparcos parallaxes and $V$ magnitudes. The masses were obtained from interpolation in grids of evolutionary tracks using the effective temperatures, luminosities, and metallicities. Finally, the metallicities were determined from a differential analysis relative to the Sun using $\mathrm{Fe}_{\mathrm{I}}$ and $\mathrm{Fe}$ II lines.

The 291 stars from Ghezzi et al. (2010a, 2010b) and 18 from del Peloso et al. (2005a, 2005b) compose the final calibration sample used in this work. Although the two subsamples were analyzed with somewhat different methods, we find that the resulting parameters are consistent. For the nine stars in common, the average differences are (in the sense Ghezzi-del Peloso): $\Delta T_{\text {eff }}=-4 \pm 41 \mathrm{~K}, \Delta[\mathrm{Fe} / \mathrm{H}]=-0.04 \pm 0.04$ dex, and $\Delta \log g=$ $0.00 \pm 0.12$ dex. In this paper, the spectra and parameters from Ghezzi et al. (2010a, 2010b) were adopted for these nine stars. We note that these parameters are consistent with those from many other catalogs of atmospheric parameters available in the literature (see, e.g., Table 5 of Ghezzi et al. 2010b).

To ensure consistency between the calibration and MARVELS survey data (the latter is described in Section 2.2), the FEROS spectra were degraded and resampled to the MARVELS resolution and sampling. The $\operatorname{IRAF}^{20}$ tasks gauss (with a value of 8.0 for the sigma parameter) and dispcor (with a value of 0.154 for the $d w$ parameter) were used, respectively, to accomplish this. The spectra were also trimmed with IRAF's task

20 IRAF (Image Reduction and Analysis Facility) is distributed by the National Optical Astronomy Observatories (NOAO), which is operated by the Association of Universities for Research in Astronomy, Inc. (AURA) under cooperative agreement with the National Science Foundation (NSO). splot in order to keep only the region 5100-5590 $\AA$, which is present in the majority of the MARVELS spectra.

\subsection{Validation Sample}

The validation sample consists of the full set of 30 stars that currently have both low-resolution MARVELS spectra and precise atmospheric parameters resulting from the analysis of highresolution spectra. Sixteen of these stars are MARVELS targets with detected companions, which were, or are, currently being subjected to more detailed analyses. ${ }^{21}$ Their high-resolution spectra were obtained with the ARC Echelle Spectrograph (Wang et al. 2003) attached to the Astrophysical Research Consortium (ARC) $3.5 \mathrm{~m}$ telescope at the Apache Point Observatory (APO; New Mexico, USA) and/or the FEROS spectrograph attached to the MPG/ESO $2.2 \mathrm{~m}$ telescope. The resolutions are $\sim 31,500$ and $\sim 48,000$, respectively, and all spectra have $S / N \gtrsim$ 100 per resolution element. Their atmospheric parameters were derived following the method described in Wisniewski et al. (2012) and are given in Table 1. Briefly, they were derived from two independent pipelines, both based on the technique of excitation and ionization equilibria of Fe I and Fe II lines. Given the consistency of the results, the two sets of parameters were combined through a weighted average, using the internal uncertainties from each pipeline as the weights.

The other 14 stars are known planet hosts, which were used in the MARVELS survey as reference objects for the RV determinations. Their atmospheric parameters are the arithmetic averages of the values taken from multiple sources in the literature (see Table 2). In this table, the uncertainties correspond to the standard deviations of the average values from the literature (and not to internal errors of the method, as in Table 1). The distribution of parameters for the 30 stars in the validation sample is also presented in Figure 1 (filled squares).

The low resolution spectra for the validation sample were obtained, as part of the survey, with the MARVELS instrument (Ge et al. 2008, 2009; Ge \& Eisenstein 2009) coupled to the SDSS $2.5 \mathrm{~m}$ telescope at APO (Gunn et al. 2006). The MARVELS instrument is a 60-object, fiber-fed, Dispersed Fixed

\footnotetext{
21 The MARVELS candidate MC10 (TYC 3010-1494-1; Mack et al. 2013) was not included in the validation sample, despite having a set of high-resolution parameters, because it is the primary component of a binary
} system with a mass ratio close to one. 
Table 2

Atmospheric Parameters of the MARVELS Reference Stars

\begin{tabular}{llccc}
\hline \hline Star & \multicolumn{1}{c}{$T_{\text {eff }}$} & {$[\mathrm{Fe} / \mathrm{H}]$} & $\log g$ & References $^{\mathrm{a}}$ \\
& $(\mathrm{K})$ & & & \\
\hline WASP 1 & $6161 \pm 52$ & $0.19 \pm 0.06$ & $4.23 \pm 0.05$ & $1,2,3$ \\
HD 4203 & $5644 \pm 61$ & $0.41 \pm 0.03$ & $4.24 \pm 0.14$ & $4,5,6,7,8,9,10,11$ \\
HD 9407 & $5661 \pm 5$ & $0.03 \pm 0.02$ & $4.45 \pm 0.03$ & $12,8,13$ \\
HD 17156 & $6057 \pm 46$ & $0.19 \pm 0.05$ & $4.20 \pm 0.11$ & $1,14,7,15,16,17$ \\
HIP 14810 & $5515 \pm 25$ & $0.27 \pm 0.02$ & $4.27 \pm 0.06$ & $18,14,5,7,19$ \\
HD 43691 & $6200 \pm 39$ & $0.29 \pm 0.02$ & $4.23 \pm 0.12$ & $14,18,7,20$ \\
HD 49674 & $5632 \pm 31$ & $0.33 \pm 0.01$ & $4.48 \pm 0.12$ & $18,7,8,9$ \\
XO-2 & $5356 \pm 19$ & $0.41 \pm 0.05$ & $4.36 \pm 0.19$ & $1,16,21$ \\
HD 68988 & $5968 \pm 48$ & $0.35 \pm 0.02$ & $4.44 \pm 0.08$ & $18,7,22,8,9,11,23$ \\
HD 80606 & $5615 \pm 70$ & $0.37 \pm 0.08$ & $4.44 \pm 0.07$ & $1,7,22,8,9,23,10,24$ \\
HD 118203 & $5767 \pm 70$ & $0.15 \pm 0.06$ & $3.92 \pm 0.04$ & $25,18,7$ \\
HAT-P-3 & $5205 \pm 28$ & $0.34 \pm 0.10$ & $4.60 \pm 0.02$ & 1,26 \\
TReS-2 & $5840 \pm 41$ & $-0.03 \pm 0.11$ & $4.39 \pm 0.09$ & $1,16,27$ \\
HAT-P-1 & $6026 \pm 71$ & $0.17 \pm 0.06$ & $4.46 \pm 0.01$ & 16,28 \\
& & & &
\end{tabular}

Notes.

a The references for each star are organized in reverse chronological order. References. (1) Torres et al. (2012); (2) Albrecht et al. (2011); (3) Stempels et al. (2007); (4) Saffe (2011); (5) Ghezzi et al. (2010b); (6) González Hernández et al. (2010); (7) Gonzalez et al. (2010); (8) Valenti \& Fischer (2005); (9) Santos et al. (2004); (10) Santos et al. (2003); (11) Laws et al. (2003); (12) Mishenina et al. (2008); (13) Mashonkina \& Gehren (2000); (14) Kang et al. (2011); (15) Barbieri et al. (2009); (16) Ammler-von Eiff et al. (2009); (17) Fischer et al. (2007); (18) Brugamyer et al. (2011); (19) Wright et al. (2009); (20) da Silva et al. (2007); (21) Burke et al. (2007); (22) Luck \& Heiter (2006); (23) Heiter \& Luck (2003); (24) Naef et al. (2001); (25) Zieliński et al. (2012); (26) Torres et al. (2007); (27) Sozzetti et al. (2007); (28) Bakos et al. (2007).

Delay Interferometer (DFDI; Wang et al. 2012a, 2012b) that outputs two fringing spectra ("beams") per object with a resolution $R \sim 12,000$ and a wavelength coverage between $\lambda \lambda \sim$ 5000-5700 A. Since each star was visited typically $\gtrsim 20$ times, the final combined spectra have an $\mathrm{S} / \mathrm{N} \gtrsim 100 \mathrm{pixel}^{-1}$ (dispersion $0.154 \AA$ pixel $^{-1}$ ). To convert the fringing spectra to conventional one-dimensional (1D) extracted spectra, the former are first preprocessed, including corrections for optical distortion and slit illumination, creating a continuum-normalized fringing spectrum. Then, for each wavelength, a sinusoid is fit to the fringing pattern, which lies perpendicular to the wavelength axis. The zero-point offset of this sinusoid is the flux value of the normalized 1D extracted spectrum for that wavelength. These fluxes (counts per pixel) are converted into flux densities (counts per unit wavelength), and then a barycentric RV correction is applied such that all 1D extracted spectra for a given star are registered to the rest wavelength.

The determination of atmospheric parameters from these lowresolution MARVELS spectra and spectral indices is described in the following sections. The ability of the method to accurately recover the high-resolution results previously derived for the MARVELS stars is regarded as the validation test of the approach presented in this paper. We note that, in spite of its relatively small size, the adopted validation sample offers the most realistic check for the quality and reliability of our method since it uses real MARVELS data (which includes instrumental effects, noise, etc.). Moreover, the set covers most regions of the parameter space defined by the calibration sample (see Figure 1) and also the ranges of atmospheric parameters expected for the whole MARVELS sample (according to the selection criteria employed during the survey target selection).

\section{DEFINITION OF THE SPECTRAL INDICES}

As already mentioned, the MARVELS resolution prevents the characterization of the stars through measurements of EWs of individual lines (and consequently classical model atmosphere analysis) because most of the spectral lines become blended with neighboring features. To overcome this problem, we have used instead an approach based on spectral indices (e.g., Worthey et al. 1994; Robinson et al. 2006), which are defined here as groups of lines formed by similar chemical species. More specifically, we have selected two groups of interest with features dominated by: (1) neutral iron-peak species (such as Fe I, VI, Cri, MnI, Co I, and Ni I) and (2) ionized species (such as Fe II, Ti II, and Cr II). These groups have, in principle, properties that should allow us to constrain $T_{\text {eff }},[\mathrm{Fe} / \mathrm{H}]$, and $\log g$. Note that we consider a feature to be dominated by a certain group of elements when their respective lines account for more than $90 \%$ of its total absorption.

The two groups were identified through a detailed inspection of the interval 5100-5590 $\AA$ on two versions of the same FEROS Ganymede spectrum (Ribas et al. 2010) that was adopted as a solar template (see Figure 2). The first version retained the original resolution $(R \sim 48,000)$ and was used in conjunction with Moore et al. (1966) to identify the individual lines that composed the indices. The second version was degraded to the MARVELS resolution and sampling (see Section 2.1) and was used to determine the initial and final wavelengths of the selected indices.

Degraded FEROS spectra of the stars HD 32147 and HD 52298 were also utilized during the selection of the indices (see Figure 3). HD 32147 is a cool metal-rich star $\left(T_{\text {eff }}=\right.$ $4850 \mathrm{~K}$ and $[\mathrm{Fe} / \mathrm{H}]=0.25$; De Silva et al. 2007) and corresponds to an extreme example of a spectrum with very strong indices. The analysis of its spectrum allowed us to refine the wavelength intervals previously defined for the indices (based on the solar spectrum) and to eliminate those that were not sufficiently isolated (i.e., did not have clear apparent continuum regions between them and the neighboring features). HD 52298 is a hot metal-poor star $\left(T_{\text {eff }}=6253 \mathrm{~K}\right.$ and $[\mathrm{Fe} / \mathrm{H}]=-0.31$; del Peloso et al. 2005a) and illustrates the opposite case, in which the strengths of the indices become too weak, preventing accurate measurements of their EWs. In summary, these two stars probe the extremes of the anticipated absorption strengths of the spectral indices and helped us to exclude potentially problematic features.

Following the procedure described above, a total of 96 potential indices were selected: 80 dominated by neutral ironpeak species and 16 by ionized species. The properties of these indices are listed in Table 3 . We have referred to them as potential indices because their actual sensitivities to the atmospheric parameters are only analyzed in Section 4.3. The final list with the best available indices for the subsequent characterization of the MARVELS sample resulted from this analysis.

\section{THE SPECTRAL INDICES METHOD}

The MARVELS sample contains $\sim 3300$ stars with numbers of visits high enough for robust planet detection, and the time required to manually analyze all objects, each with $\gtrsim 40$ spectra, would be prohibitively large. Moreover, such an approach would be more error prone due to subjective choices that are inevitably made throughout the analysis of stellar spectra (e.g., during the continuum normalization). To avoid these issues, the 


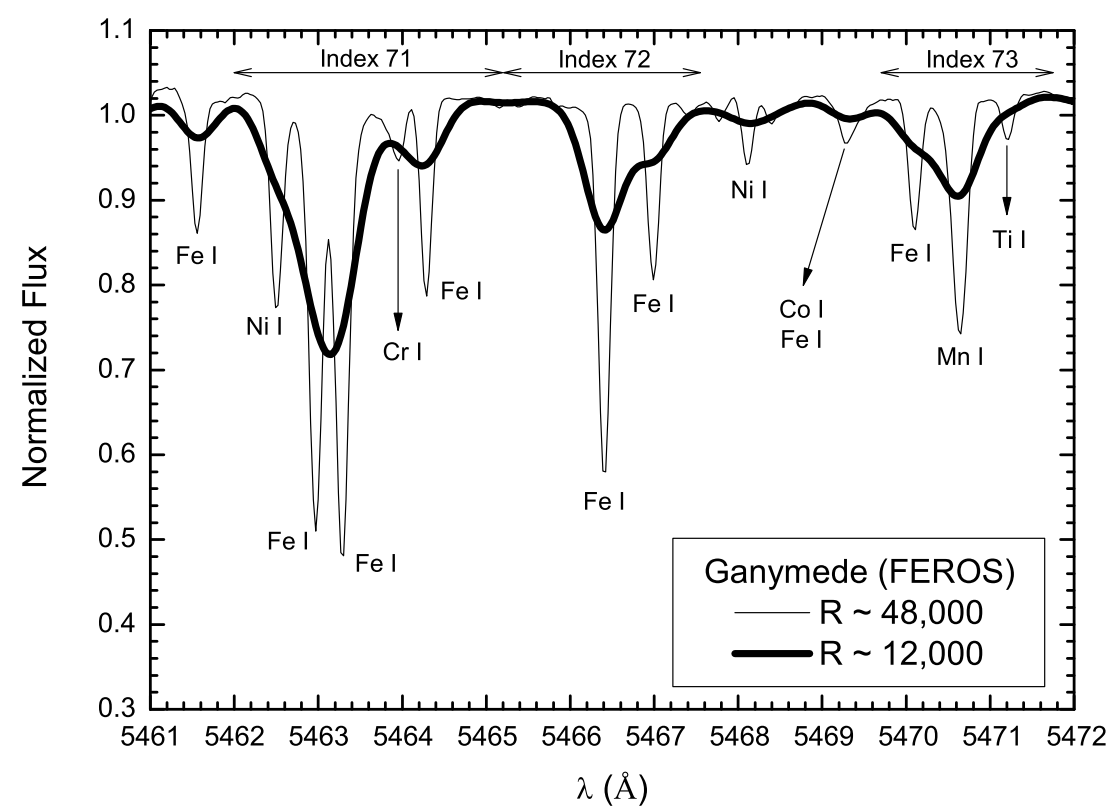

Figure 2. Section of the FEROS Ganymede spectrum used as a solar template. The thin line shows the spectrum at its original resolution $(R \sim 48,000)$ and the thick line represents the version that was degraded to the MARVELS resolution $(R \sim 12,000)$. The most prominent individual lines are identified by their chemical species. Three spectral indices dominated by neutral iron-peak species and their respective wavelength intervals are shown as examples of the selection procedure. The other indices are distributed throughout the full wavelength range used in the analysis (5100-5590 $)$.

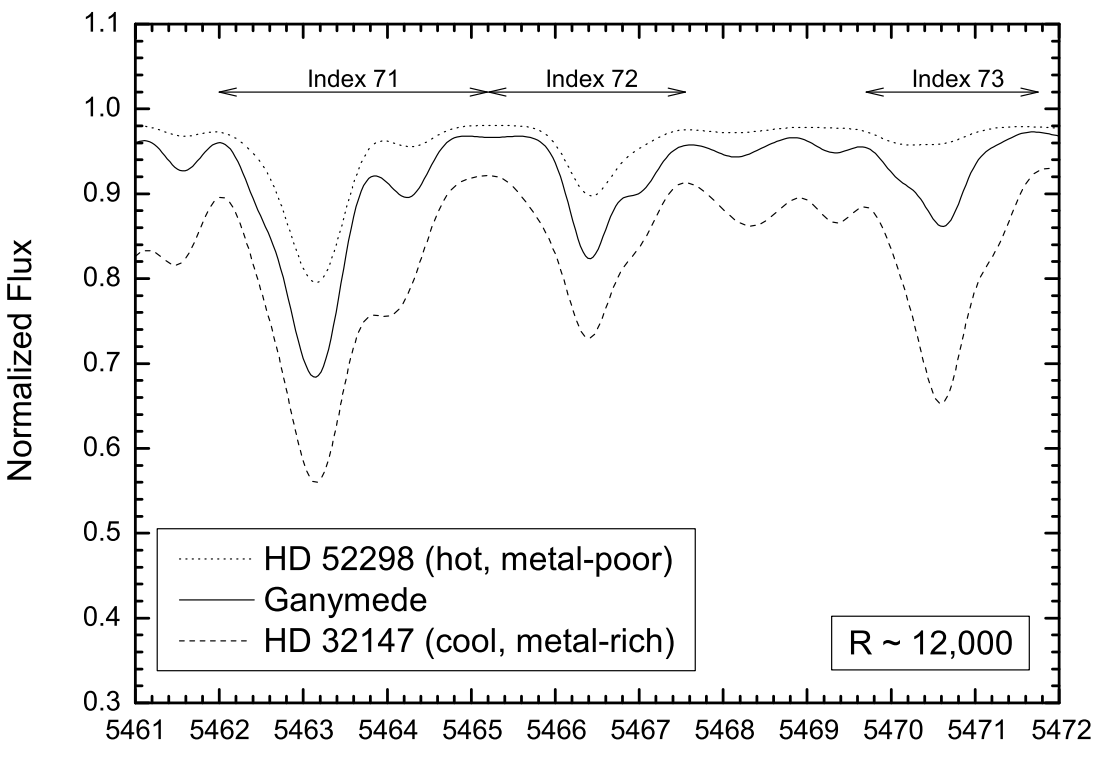

$\lambda(\AA)$

Figure 3. Section of the FEROS spectra of HD 52298 (dotted line), Ganymede (solid line), and HD 32147 (dashed line). They were all degraded to the MARVELS resolution $(R \sim 12,000)$ and the continua have been shifted vertically for clarity. The three spectral indices shown as examples are the same as in Figure 2 . It is clear that their definitions are appropriate for the three stars, though their parameters are significantly different.

determination of the atmospheric parameters was automatized through the development of three codes, which will be made publicly available through the Brazilian Participation Group Scientific Portal. ${ }^{22}$ These codes, as well as the tests performed to ascertain their quality, are described in more detail in the following sections.

\subsection{Normalization}

The normalization of a spectrum is a time-consuming task since one must test several parameters for choosing the curve that best describes the continuum of a particular star. Because

\footnotetext{
22 http://bpg.linea.gov.br/
}

we aim to apply the indices technique for large stellar samples (in particular, from the MARVELS survey), an automatic tool to perform this task is required. To this end, the first program was developed to fit a curve to and normalize the continuum on the spectra of solar-type stars. It was inspired by the task continuum from IRAF, but has the advantage of automatically testing many different normalizations and determining which one provides the best fit. This feature turns the normalization process into a fast, non-interactive, and systematic procedure, characteristics that are essential for the analysis of large samples of stars.

The code uses as input the reduced, defringed, 1D Dopplercorrected spectra (in FITS format) that are given as part of 
Table 3

Properties of the Spectral Indices

\begin{tabular}{|c|c|c|c|c|}
\hline Index & $\begin{array}{c}\lambda_{i} \\
(\AA)\end{array}$ & $\begin{array}{l}\lambda_{f} \\
(\AA)\end{array}$ & Dominant Lines & Notes \\
\hline 1 & 5100.40 & 5102.00 & Fe II & 2 \\
\hline 2 & 5102.00 & 5104.90 & Ni I, Fe I & 3 \\
\hline 3 & 5105.10 & 5106.05 & $\mathrm{Cu} \mathrm{I}$ & 3 \\
\hline 4 & 5106.90 & 5108.25 & $\mathrm{Fe}_{\mathrm{I}}$ & 3 \\
\hline 5 & 5109.95 & 5111.05 & $\mathrm{Fe} I$ & 3 \\
\hline 6 & 5130.95 & 5132.20 & Fe I, Ni I & 3 \\
\hline 7 & 5135.55 & 5137.90 & Fe I, Ni I & 3 \\
\hline 8 & 5138.05 & 5140.45 & Fe I, Cr I & 1 \\
\hline 9 & 5140.65 & 5144.15 & Fe I, Ni I & 1 \\
\hline 10 & 5194.35 & 5196.95 & Fe I, Cr I, Mn I & 1 \\
\hline 11 & 5196.95 & 5198.00 & Fe II, Fe I, Ni I & 1 \\
\hline 12 & 5198.25 & 5199.35 & $\mathrm{Fe}_{\mathrm{I}}$ & 1 \\
\hline 13 & 5201.55 & 5203.30 & $\mathrm{Fe}_{\mathrm{I}}$ & 1 \\
\hline 14 & 5203.30 & 5209.45 & $\mathrm{Cr}$ I, Fe I & 1 \\
\hline 15 & 5213.55 & 5216.90 & $\mathrm{Fe}_{\mathrm{I}}$ & 1 \\
\hline 16 & 5229.10 & 5230.95 & $\mathrm{Fe}_{\mathrm{I}}$ & 1 \\
\hline 17 & 5231.85 & 5233.70 & $\mathrm{Fe}_{\mathrm{I}}$ & 1 \\
\hline 18 & 5234.05 & 5236.75 & $\mathrm{Fe}$ II, Fe I & 1 \\
\hline 19 & 5236.75 & 5238.00 & Cr II & 1 \\
\hline 20 & 5241.25 & 5244.85 & $\mathrm{Fe}_{\mathrm{I}}$ & 1 \\
\hline 21 & 5249.75 & 5254.25 & $\mathrm{Fe} I$ & 1 \\
\hline 22 & 5254.25 & 5256.35 & Cr I, Fe I, Mn I & 1 \\
\hline 23 & 5272.60 & 5274.05 & $\mathrm{Fe}_{\mathrm{I}}$ & 1 \\
\hline 24 & 5274.05 & 5276.80 & Fe II, Fe I, Cr I, Cr II & 1 \\
\hline 25 & 5279.35 & 5280.95 & Cr I, Fe I, Cr II & 1 \\
\hline 26 & 5280.95 & 5282.85 & $\mathrm{Fe}_{\mathrm{I}}$ & 1 \\
\hline 27 & 5282.85 & 5286.65 & Fe I, Ti I, Fe II & 1 \\
\hline 28 & 5288.00 & 5289.10 & $\mathrm{Fe}_{\mathrm{I}}$ & 1 \\
\hline 29 & 5296.15 & 5299.45 & $\mathrm{Cr}, \mathrm{Fe}_{\mathrm{I}}$ & 1 \\
\hline 30 & 5301.50 & 5303.25 & Fe I & 1 \\
\hline 31 & 5306.75 & 5308.00 & $\mathrm{Fe}_{\mathrm{I}}$ & 1 \\
\hline 32 & 5308.00 & 5309.45 & Cr II & 1 \\
\hline 33 & 5312.10 & 5314.25 & Cr II, Cr I & 4 \\
\hline 34 & 5314.25 & 5315.65 & $\mathrm{Fe}_{\mathrm{I}}$ & 4 \\
\hline 35 & 5315.90 & 5317.90 & Fe II & 1 \\
\hline 36 & 5320.50 & 5322.65 & Fe I & 1 \\
\hline 37 & 5322.65 & 5325.00 & $\mathrm{Fe}_{\mathrm{I}}$ & 1 \\
\hline 38 & 5327.05 & 5330.85 & Fe I, Cr I & 1 \\
\hline 39 & 5332.05 & 5333.55 & Fe I, V II & 4 \\
\hline 40 & 5334.55 & 5335.60 & Cr II, Co I & 4 \\
\hline 41 & 5336.30 & 5337.35 & Ti II & 2 \\
\hline 42 & 5338.90 & 5341.95 & Fe I, Cr I, Mn I & 1 \\
\hline 43 & 5341.95 & 5344.05 & $\mathrm{CoI}, \mathrm{Fe} \mathrm{I}$ & 1 \\
\hline 44 & 5345.10 & 5346.40 & Cr I & 1 \\
\hline 45 & 5347.35 & 5348.90 & CrI & 1 \\
\hline 46 & 5352.65 & 5354.20 & Fe I, Co I, Ni I & 1 \\
\hline 47 & 5360.70 & 5362.15 & $\mathrm{Fe}_{\mathrm{I}}$ & 1 \\
\hline 48 & 5362.20 & 5363.65 & Fe II, Fe I, Co I & 1 \\
\hline 49 & 5363.65 & 5366.05 & $\mathrm{Fe}_{\mathrm{I}}$ & 1 \\
\hline 50 & 5366.90 & 5368.15 & $\mathrm{Fe}_{\mathrm{I}}$ & 1 \\
\hline 51 & 5368.95 & 5372.85 & Fe I, Ni I, Co I & 1 \\
\hline 52 & 5373.10 & 5374.70 & $\mathrm{Fe}_{\mathrm{I}}$ & 1 \\
\hline 53 & 5377.10 & 5378.55 & Mn I & 1 \\
\hline 54 & 5382.65 & 5384.35 & $\mathrm{Fe}_{\mathrm{I}}$ & 4 \\
\hline 55 & 5385.85 & 5388.00 & Fe I, Cr I & 4 \\
\hline 56 & 5388.85 & 5392.30 & $\mathrm{Fe}_{\mathrm{I}}$ & 1 \\
\hline 57 & 5392.50 & 5394.05 & $\mathrm{Fe}_{\mathrm{I}}$ & 1 \\
\hline 58 & 5394.05 & 5395.75 & $\mathrm{Mn}_{\mathrm{I}}, \mathrm{Fe} \mathrm{I}$ & 1 \\
\hline 59 & 5395.75 & 5398.95 & $\mathrm{Fe}_{\mathrm{I}}$ & 1 \\
\hline 60 & 5399.95 & 5402.05 & $\mathrm{Fe}$ I, Cr I & 1 \\
\hline 61 & 5403.20 & 5407.00 & $\mathrm{Fe}_{\mathrm{I}}$ & 4 \\
\hline 62 & 5408.35 & 5411.80 & Cr I, Fe I, Ni I & 4 \\
\hline 63 & 5414.45 & 5416.00 & $\mathrm{Fe}_{\mathrm{I}}$ & 1 \\
\hline 64 & 5416.50 & 5417.60 & $\mathrm{Fe}_{\mathrm{I}}$ & 2 \\
\hline 65 & 5417.65 & 5419.60 & Ti II & 2 \\
\hline
\end{tabular}

Table 3

(Continued)

\begin{tabular}{|c|c|c|c|c|}
\hline Index & $\begin{array}{c}\lambda_{i} \\
(\AA)\end{array}$ & $\begin{array}{l}\lambda_{f} \\
(\AA)\end{array}$ & Dominant Lines & Notes \\
\hline 66 & 5422.90 & 5425.65 & $\mathrm{Fe}_{\mathrm{I}}, \mathrm{Ni}$ I, Fe II & 4 \\
\hline 67 & 5427.30 & 5431.00 & Fe I & 4 \\
\hline 68 & 5431.90 & 5435.35 & $\mathrm{Fe}$ I, Mn I & 1 \\
\hline 69 & 5435.35 & 5437.60 & Ni I, Fe I & 1 \\
\hline 70 & 5454.30 & 5456.95 & $\mathrm{Fe}_{\mathrm{I}}$ & 1 \\
\hline 71 & 5462.00 & 5465.20 & Fe I, Ni I & 1 \\
\hline 72 & 5465.20 & 5467.55 & $\mathrm{Fe}_{\mathrm{I}}$ & 1 \\
\hline 73 & 5469.70 & 5471.75 & $\mathrm{Mn}$ I, Fe I & 1 \\
\hline 74 & 5475.65 & 5477.45 & $\mathrm{Ni}$ I, Fe I & 1 \\
\hline 75 & 5482.65 & 5484.25 & Fe I, Co I & 1 \\
\hline 76 & 5486.35 & 5488.60 & Fe I & 1 \\
\hline 77 & 5496.80 & 5498.50 & Fe I & 1 \\
\hline 78 & 5500.20 & 5502.45 & $\mathrm{Fe}_{\mathrm{I}}$ & 1 \\
\hline 79 & 5505.10 & 5507.55 & $\mathrm{Fe}$ I, Mn I & 1 \\
\hline 80 & 5516.00 & 5517.75 & $\mathrm{Mn}$ I, Fe I & 1 \\
\hline 81 & 5521.85 & 5522.95 & $\mathrm{Fe}_{\mathrm{I}}$ & 1 \\
\hline 82 & 5524.70 & 5526.15 & $\mathrm{Fe} I$ & 1 \\
\hline 83 & 5531.45 & 5533.80 & $\mathrm{Fe} I$ & 1 \\
\hline 84 & 5534.05 & 5536.15 & Fe I, Ba I, Fe II & 1 \\
\hline 85 & 5542.30 & 5544.65 & $\mathrm{Fe}_{\mathrm{I}}$ & 3 \\
\hline 86 & 5545.50 & 5547.85 & $\mathrm{Fe}_{\mathrm{I}}, \mathrm{V}_{\mathrm{I}}$ & 3 \\
\hline 87 & 5552.90 & 5555.70 & Fe I, Ni I & 3 \\
\hline 88 & 5559.40 & 5560.85 & $\mathrm{Fe}_{\mathrm{I}}$ & 3 \\
\hline 89 & 5562.05 & 5564.50 & $\mathrm{Fe}_{\mathrm{I}}$ & 3 \\
\hline 90 & 5564.85 & 5566.45 & $\mathrm{Fe}_{\mathrm{I}}$ & 3 \\
\hline 91 & 5566.80 & 5568.20 & $\mathrm{Fe}_{\mathrm{I}}$ & 3 \\
\hline 92 & 5568.80 & 5570.30 & $\mathrm{Fe}_{\mathrm{I}}$ & 3 \\
\hline 93 & 5571.95 & 5574.00 & $\mathrm{Fe}_{\mathrm{I}}$ & 3 \\
\hline 94 & 5575.40 & 5576.80 & $\mathrm{Fe}_{\mathrm{I}}$ & 3 \\
\hline 95 & 5577.90 & 5579.25 & $\mathrm{Ni}$ I & 3 \\
\hline 96 & 5585.85 & 5587.35 & $\mathrm{Fe}_{\mathrm{I}}$ & 3 \\
\hline
\end{tabular}

Notes. (1) Indices used in the final analysis. (2) Indices removed because their calibrations had $R^{2}<0.9$ (see Section 4.3). (3) Indices excluded because they were not present in all 120 solar exposures (see Section 5.2.1). (4) Indices eliminated because $\left|\left\langle\mathrm{EW}_{\text {MARVELS }}\right\rangle-\mathrm{EW}_{\mathrm{FEROS}}\right|>\sigma\left(\left\langle\mathrm{EW}_{\mathrm{MARVELS}}\right\rangle\right)$ in the solar spectra (see Section 5.2.1).

the final products delivered by the MARVELS pipeline. It is possible to combine more than one spectrum using the median counts as weights and cosmic rays can be removed through a $\sigma$ clipping algorithm if at least five spectra are being coadded. A number of 1D Legendre polynomials are then fit to the continuum points of the individual or combined spectra, in a wavelength range defined by the user. Both the number of polynomials tested and of points considered in the fit depend on an additional set of input parameters provided by the user: polynomial order, high and low rejection limits (as well as the steps with which they are changed), grow parameter, and number of iterations to be performed. All parameters have the same definitions as in the task continuum from IRAF.

The program computes as many solutions as the choice of the input parameters described above, but testing all possible combinations for each star would require an excessive amount of time and most of the solutions would not be considered satisfactory. Thus, we decided to restrict the set of input parameters using 27 stars (7 with degraded FEROS spectra and 20 MARVELS targets with spectra from the survey) with parameters that sample the range of indices strengths expected to be found in the stars from the MARVELS survey. We note that this test set included cool metal-rich stars to ensure that suitable apparent continuum regions could still be found, allowing accurate normalizations. The best visual normalizations were 


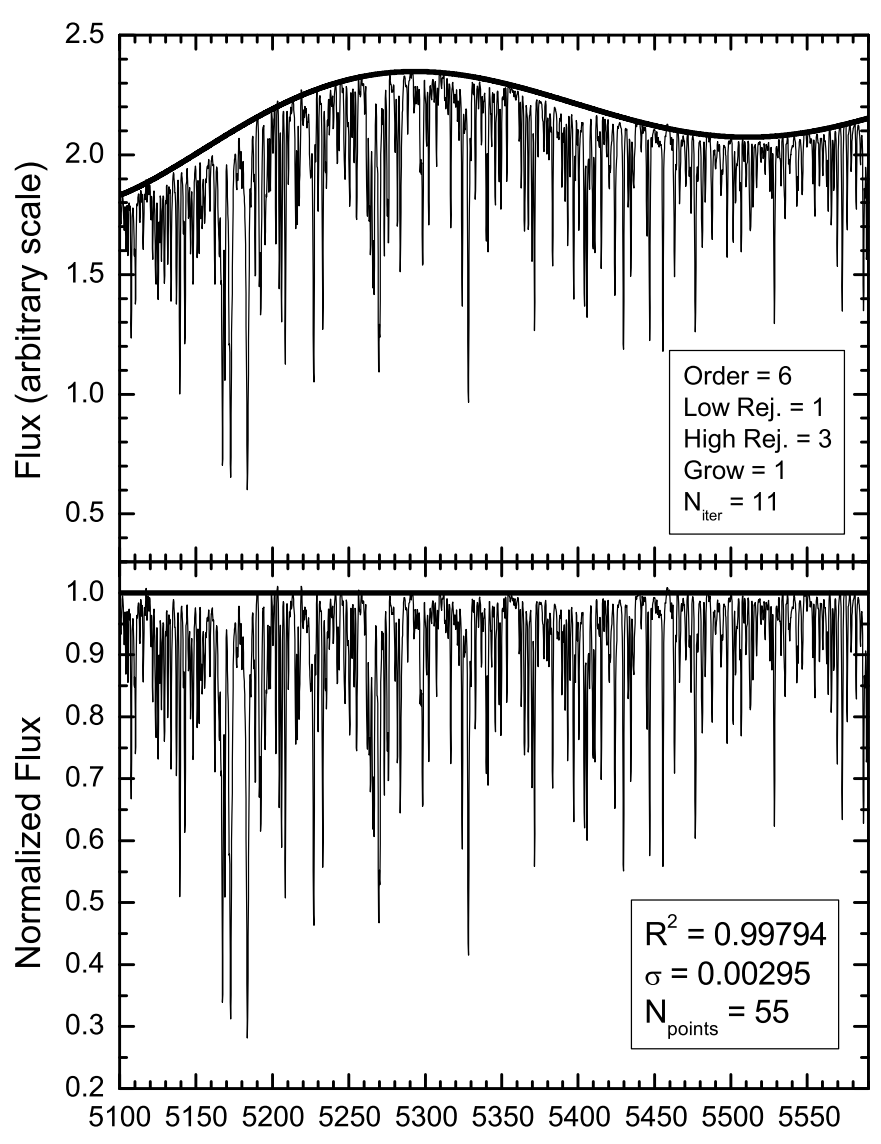

$\lambda(\AA)$

Figure 4. Example of the normalization procedure. Upper panel: Degraded FEROS spectrum of the calibration star HD 20630 before the normalization. The thick black line represents the polynomial that best fits the continuum. The values of the polynomial order, low and high rejection limits, grow parameter, and number of iterations are provided. Lower panel: Same spectrum after the continuum normalization. The black thick line now shows the normalized continuum level at 1.0. The correlation coefficient $R^{2}$, standard deviation $\sigma$, and number of points of the fit are also given.

found for the following values of the input parameters: five or six for the order of the Legendre polynomials; $1.0-1.5$ and 3.0-4.0 (both with steps of 0.5 ) for the low and high rejection parameters; one for the grow parameter; 11 for the number of iterations. It was also observed that the fits with the highest correlation coefficient $R^{2}$ always produced the best results (the same was not true for the cases with the lowest values of the standard deviation of the residuals $\sigma$ ) and this is the reason why we chose this statistical criterion to select the final solutions.

There are some limitations that could not be circumvented by the code after these restrictions were made. These issues are mainly related to the inability to remove cosmic rays (due to inefficient clipping or an insufficient number of spectra to be combined) or regions with defects (e.g., strong curvatures on the edges). The former can be minimized by combining a larger number of spectra for each star (if available, which was not the case for the stars with FEROS data). The second limitation is more complicated, but the usage of higher values for the polynomial order proved to be effective in most of the cases.

The spectra of the 309 stars from the calibration sample (Section 2.1) were normalized using the code, restrictions, and criteria described above. An example of the normalization procedure is shown in Figure 4. We visually inspected the final solutions for each of the 309 stars to search for normalization problems that could undermine the indices calibrations. The solutions provided by the code had to be replaced by visually better fits in $9 \%$ of the cases, which defines a reasonable limit to the degree of efficient automation of the normalization process. For the analysis of the complete MARVELS sample, the number of spectra will be larger by a factor of $\sim 100$ and such a visual inspection will not be feasible. We are currently working on a complementary code to automatically identify these cases that require human intervention based on large variations of the continuum fit parameters for individual spectra of a given star or the measured EWs for some indices.

Even though an effort was made to ensure that the continuum placement was as accurate as possible, it is possible that small offsets are still present for a few spectra. The typical $\mathrm{S} / \mathrm{N}$ of the individual MARVELS spectra for the validation sample is $\sim 100$, which corresponds to an rms error of the continuum of $\sim(\mathrm{S} / \mathrm{N})^{-1} \sim 0.01$. Using the method described in Sections $4.2,4.3$, and 4.4 as well as the final results presented in Section 5.2.2, we have checked that an offset of $\pm 1 \%$ in the continuum placement causes the following average variations on the derived atmospheric parameters: $\Delta T_{\text {eff }}=-5 \pm 42 \mathrm{~K}$, $\Delta[\mathrm{Fe} / \mathrm{H}]= \pm 0.12 \pm 0.04$, and $\Delta \log g=\mp 0.07 \pm 0.07$. Note that these values correspond to the unlikely case in which a global systematic offset would shift all the spectra of all the stars in the sample in the same direction and by the same amount. Therefore, they should be regarded as the maximum possible errors that could be introduced by normalization issues. The actual errors will therefore be smaller than estimated here (see also discussion in Section 5.2.2).

\subsection{Measurements of the Equivalent Widths of the Indices}

The normalized spectra obtained above for the calibration stars were used as input for the next code, which measures the EWs of a list of indices by direct integration of their profiles. This method was chosen because most of the indices are formed by multiple lines with different profiles. The integrations were performed in the wavelength intervals defined for each index (see Table 3) adopting the value of 1.0 for the normalized continuum flux.

This code was used to measure the EWs of the 96 indices (see Section 3) in the degraded FEROS spectra of all 309 stars from the calibration sample. We have checked that all indices' EWs could be correctly measured even for the most metal-poor stars in the sample. A quick look at columns "EWmin" in Table 4 and "Notes" in Table 3 reveals that $4.81 \mathrm{~m} \AA$ is the lowest value for a EW used in the calibrations that were adopted for the derivation of the atmospheric parameters (more details about this selection are given in the next section). This value occurs for the star HD $76932\left(T_{\text {eff }}=5850 \mathrm{~K}\right.$ and $\left.[\mathrm{Fe} / \mathrm{H}]=-0.84\right)$ in the calibration for index 80 and is consistent with the lower limit of the measuring capabilities of our automated software.

Problematic EWs were identified and discarded during the construction of the regressions (see Section 4.3), since bad measurements appeared as clear outliers relative to the general trends observed. For the MARVELS targets, multiple spectra from different visits are available for a given star and the outliers can be excluded by a simple $\sigma$ clipping procedure.

\subsection{Construction of the Calibrations}

The previous section discussed the measurements of the EWs of the 96 spectral indices in the spectra of the calibration stars. It was also mentioned in Section 2.1 that all these objects have 
Table 4

Calibrations of the Indices

\begin{tabular}{|c|c|c|c|c|c|c|c|c|c|c|c|c|c|c|c|}
\hline$\overline{\text { Index }}$ & $c_{0}$ & $c_{1}$ & $c_{2}$ & $c_{3}$ & $c_{4}$ & $c_{5}$ & $c_{6}$ & $c_{7}$ & $c_{8}$ & $c_{9}$ & $\overline{R^{2}}$ & $\begin{array}{c}\sigma \\
(\mathrm{mA})\end{array}$ & $\mathrm{N}$ & $\begin{array}{c}\mathrm{EW}_{\min } \\
(\mathrm{m} \AA)\end{array}$ & $\begin{array}{c}\mathrm{EW}_{\max } \\
(\mathrm{m} \AA)\end{array}$ \\
\hline 1 & $5 E+03$ & $E+02$ & $1.04 \mathrm{E}+00$ & 2 & 2 & $4.34 \mathrm{E}+01$ & $-5.57 \mathrm{E}-02$ & $5.88 \mathrm{E}+01$ & 34 & 1 & 895 & 11.55 & 261 & 1.65 & 230.01 \\
\hline 2 & $55 \mathrm{E}+02$ & $4 \mathrm{E}+02$ & $5.20 \mathrm{E}-01$ & $3 \mathrm{E}+02$ & $1.97 \mathrm{E}-02$ & $1.36 \mathrm{E}+02$ & $-2.72 \mathrm{E}-02$ & $39 \mathrm{E}+01$ & 4.35E-05 & $-5.49 \mathrm{E}+01$ & 926 & 18.39 & 264 & .01 & 02.49 \\
\hline 3 & $\mathrm{E}+02$ & +02 & $\sigma L$ & $3 \mathrm{E}+02$ & $1.94 \mathrm{E}-02$ & $4.35 \mathrm{E}+01$ & & $7.23 E+00$ & 1E-06 & & & 6.06 & 71 & & 166.78 \\
\hline 4 & $2 \mathrm{E}+03$ & $8 \mathrm{E}+02$ & 7.82E- 01 & $48 \mathrm{E}+02$ & $-4.32 \mathrm{E}-02$ & $-1.31 \mathrm{E}+01$ & $-5.91 \mathrm{E}-02$ & & $8.09 \mathrm{E}-05$ & & 0.974 & 7.48 & 266 & 143.32 & 57.77 \\
\hline 5 & $88 \mathrm{E}+03$ & $54 \mathrm{E}+02$ & $-5.41 \mathrm{E}-01$ & $7 \mathrm{E}+02$ & $4.75 \mathrm{E}-02$ & $-1.74 \mathrm{E}+01$ & $-3.68 \mathrm{E}-02$ & $7.28 \mathrm{E}+00$ & $5.32 \mathrm{E}-05$ & $9.16 \mathrm{E}+00$ & 0.980 & 5.56 & 266 & 94.09 & 20.62 \\
\hline 6 & $1.24 \mathrm{E}+03$ & $42 \mathrm{E}+02$ & $-2.62 \mathrm{E}-01$ & $-8.39 \mathrm{E}+01$ & $3.16 \mathrm{E}-02$ & $-5.26 \mathrm{E}+01$ & $-2.75 \mathrm{E}-02$ & $-7.08 \mathrm{E}+00$ & $2.87 \mathrm{E}-05$ & $2.75 \mathrm{E}+01$ & 0.955 & 6.90 & 267 & 55.36 & 50.87 \\
\hline 7 & $3.60 \mathrm{E}+03$ & +02 & . & $1.65 \mathrm{E}+02$ & 2 & $.65 \mathrm{E}+01$ & $-8.45 \mathrm{E}-02$ & $2.62 \mathrm{E}+01$ & $1.20 \mathrm{E}-04$ & $3.87 \mathrm{E}+01$ & 0.964 & 13.58 & 270 & & 1.45 \\
\hline 8 & $1 \mathrm{E}+03$ & $36 \mathrm{E}+02$ & $2.94 \mathrm{E}+00$ & $9.48 \mathrm{E}+02$ & $6.03 \mathrm{E}-02$ & $4.85 \mathrm{E}+01$ & $-1.47 \mathrm{E}-01$ & $-5.02 \mathrm{E}+01$ & $2.85 \mathrm{E}-04$ & & 0.986 & 15.23 & 266 & & 56.46 \\
\hline 9 & $8.65 \mathrm{E}+03$ & & $2.87 \mathrm{E}+00$ & & $3.08 \mathrm{E}-02$ & $-8.81 \mathrm{E}+01$ & $-1.49 \mathrm{E}-01$ & $-5.27 \mathrm{E}+01$ & $2.83 \mathrm{E}-04$ & $6.37 \mathrm{E}+01$ & 0.975 & 20.20 & 266 & 3.52 & 1006.07 \\
\hline 10 & $5.71 \mathrm{E}+03$ & +02 & $-2.22 \mathrm{E}+00$ & $7.34 \mathrm{E}+02$ & $-2.53 \mathrm{E}-02$ & $2.64 \mathrm{E}+01$ & $-2.11 \mathrm{E}-01$ & & & $6.17 \mathrm{E}+01$ & 0.985 & 13.55 & 268 & .55 & 851.70 \\
\hline 11 & $9.15 \mathrm{E}+02$ & & $2.32 \mathrm{E}-01$ & $-4.08 \mathrm{E}+01$ & $1.82 \mathrm{E}-02$ & $-2.51 \mathrm{E}+01$ & $-3.08 \mathrm{E}-02$ & $.12 \mathrm{E}+00$ & & $2.26 \mathrm{E}+01$ & 0.946 & 4.75 & 266 & 58.48 & 76.57 \\
\hline 12 & $\mathrm{LT}$ & & S.1 & 22 & $\ldots$ & $\cdots$ & -9.011 & -1.30 & & $3.38 \mathrm{E}$ & 0.966 & 5.93 & 72 & & 2.45 \\
\hline 13 & $E+03$ & & 00 & & $\ldots$ & . & $-1.15 \mathrm{E}-01$ & & & $2.24 \mathrm{E}+01$ & 0.976 & 9.78 & & & 24.25 \\
\hline 14 & & & -1.3 & & $2.32 \mathrm{E}-01$ & $2.90 \mathrm{E}+02$ & -7.72 & & & & 0.988 & 46.94 & 63 & & \\
\hline 15 & $4.08 \mathrm{E}+03$ & & -1.76 & & $-1.21 \mathrm{E}-01$ & $4.68 \mathrm{E}+01$ & $-1.85 \mathrm{I}$ & $7.75 \mathrm{E}+$ & & $.38 \mathrm{E}+01$ & 0.985 & 13.75 & 50 & & 773.83 \\
\hline 16 & & & 1.1 & & 0.1 & & -8.2 & 1.60 & & & 0.987 & 05 & 56 & & 60.47 \\
\hline 17 & +03 & 02 & -1.3 & & 7.87 & $1.13 \mathrm{E}+02$ & -1.5 & $-1.23 \mathrm{E}+01$ & & $-4.58 \mathrm{E}+01$ & 991 & 9.96 & 52 & 57 & 3.59 \\
\hline 18 & 502 & & -2.6 & -02 & 2 & $-1.87 \mathrm{E}+01$ & -2.9 & +01 & & +01 & .982 & 8.22 & 271 & & 3.52 \\
\hline 19 & & & -1.6 & -2.0 & 3 & $-1.00 \mathrm{E}+01$ & -7.3 & & & $2.66 \mathrm{E}+01$ & 0.934 & 3.45 & 269 & 07 & 9.20 \\
\hline 20 & & & 30 & & & & & & & & 0.968 & 9.63 & 265 & 92 & 400.24 \\
\hline 21 & & & 2.1 & 22 & 7.50 & $63 \mathrm{E}+$ & -3.08 & 1.00 & & $.10 \mathrm{E}+01$ & 0.987 & 13.15 & 69 & 183.32 & 46.77 \\
\hline 22 & & & $-12+3$ & 02 & 04 & & & & & & .991 & 7.21 & 68 & & 11.35 \\
\hline 23 & & & -6.5 & & -3.2 & $3.88 \mathrm{E}+01$ & -6.5 & & & & 0.988 & 5.30 & 267 & & 3.42 \\
\hline 24 & & & $-84>-$ & & 416 & & -4.8 & & & $1.84 \mathrm{E}$ & 0.986 & 10.74 & 71 & & 67.33 \\
\hline 25 & & & 3. & & & 1 & & & & & & & & & .59 \\
\hline 26 & & & -1.2 & & 8.4 & -01 & -6.5 & & & $1.43 \mathrm{~F}$ & 990 & 7.13 & 62 & & 17.87 \\
\hline 27 & & & $11^{3}$ & & & $9.46 \mathrm{E}+01$ & -5 . & & & +01 & .989 & 10.87 & 54 & & 2.70 \\
\hline 28 & 1. & & -26 & -6.0 & -154 & & $9.59 \mathrm{E}-03$ & +01 & 05 & 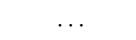 & 0.987 & 2.08 & 263 & 97 & 35.33 \\
\hline 29 & & & 2.2 & & & & & & & $8.84 \mathrm{E}-$ & 91 & & 0 & & 1148.08 \\
\hline 30 & & & -6.8 & & $-6.82 \mathrm{E}$ & 01 & -5.3 & & & & 0.987 & & 8 & 10 & 66.37 \\
\hline 31 & & & -3 & & & & & & & 1010 & 62 & 3.35 & 59 & & 50.74 \\
\hline 32 & & & 10 & 1 & $2.25 \mathrm{E}-02$ & -7.301 & & & & & & 3.51 & & & .54 \\
\hline 33 & & & $332>3$ & +01 & $32+2$ & & & & & & & 57 & & & 2.86 \\
\hline 34 & & & & & & 0.502 & & & & & & & & & .67 \\
\hline 35 & & & & & & & 6.48 & & & & 9 & & 5 & & 69.06 \\
\hline 36 & & & -3 . & & 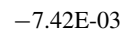 & 1 & & & & -2.22 & 76 & 5.83 & 76 & & 2.53 \\
\hline 37 & & $E+02$ & $-126 \mathrm{~F}+00$ & +03 & Q $0650 ?$ & $1.21 \mathrm{E}+02$ & $-1.24 \mathrm{E}-01$ & $2.13 \mathrm{E}+01$ & & $-4.83 \mathrm{E}+01$ & 0.988 & 11.81 & 273 & 147.63 & 96.29 \\
\hline 38 & & & 0 & & 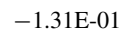 & $1.59 \mathrm{E}+02$ & -2.37 & & & & & 18.36 & 266 & & 1453.86 \\
\hline 39 & & & 4. & 0 & -2. & $\ldots$ & $\ldots$ & & & 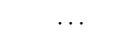 & 4 & 0 & 4 & & 5.36 \\
\hline 40 & & & $6 .(1$ & & $3.33 \mathrm{E}-\mathrm{C}$ & $.77 E+00$ & $5.47 \mathrm{E}-0$ & & & & 901 & 2.90 & 281 & & 53.33 \\
\hline 41 & & & -1.7 & 22 & $\ldots$ & $-1.32 \mathrm{E}+01$ & $\ldots$ & & & $1.01 \mathrm{E}+01$ & 0.872 & 3.24 & 282 & 60.27 & 110.59 \\
\hline 1) & & & & & $-1.13 \mathrm{E}-01$ & $1.05 \mathrm{E}+02$ & $-1.53 \mathrm{E}-01$ & & & $-3.36 \mathrm{E}+01$ & 0.990 & 00 & 71 & & 2.36 \\
\hline 43 & & & & & 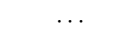 & $\ldots$ & & & & 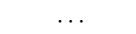 & 0 & & & & \\
\hline 44 & & & 1. & & -3.7 & & -5.8 & & & $1.58 \mathrm{E}+\mathrm{C}$ & 1 & 0 & & & 9.05 \\
\hline 45 & & & 7." & & 2 & .. & $-3.36 \mathrm{I}$ & 01 & & & 0.981 & 5.26 & 68 & 94 & 02.09 \\
\hline 46 & & & -1.2 & $-5.95 \mathrm{E}+01$ & $8.18 \mathrm{E}-03$ & $-4.45 \mathrm{E}+00$ & $-1.22 \mathrm{E}-02$ & $-6.81 \mathrm{E}+00$ & & $1.51 \mathrm{E}+01$ & 0.981 & 3.39 & 273 & 37.12 & 182.41 \\
\hline 47 & & & & & 7005 & & $2.38 \mathrm{E}-02$ & $1.71 \mathrm{E}+01$ & & & 0.977 & 3.24 & & & 41.32 \\
\hline 48 & & 1 & & & & & $\ldots$ & & 66 & & 6 & & & & 3.64 \\
\hline 49 & & & -6.1 & & $1.625>$ & $30 \mathrm{E}+01$ & $-3.15 \mathrm{E}$ & & & 01 & 980 & 7.33 & 271 & & 06.41 \\
\hline 50 & & & -4.4 & & -3.5 & $52 \mathrm{E}+01$ & $-2.57 \mathrm{E}-02$ & $5 \mathrm{E}+01$ & -05 & $-2.01 \mathrm{E}+01$ & 0.979 & 5.83 & 269 & 80.91 & 288.34 \\
\hline 51 & & & & & $-1.86 \mathrm{E}-01$ & $4 \mathrm{E}+02$ & & $32 \mathrm{E}+01$ & & $\ldots$ & 0.991 & 20.48 & 279 & 274.01 & 392.36 \\
\hline 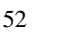 & & & & & 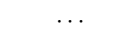 & 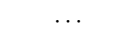 & $-1.32 \mathrm{I}$ & & & & & & & & 0.34 \\
\hline 53 & & & 9. & & $-1.94 \mathrm{I}$ & r. & $\ldots$ & & & $\ldots$ & 0.978 & 5. & 280 & 50 & 93.21 \\
\hline 54 & & & 0.1 & & -4.61 & $4.92 \mathrm{E}+01$ & $-3.77 \mathrm{E}$ & & & $-5.24 \mathrm{E}+01$ & 980 & 8.21 & 268 & 63 & 14.96 \\
\hline 55 & & & & & $-1.95 \mathrm{E}-$ & & $2.84 \mathrm{E}-02$ & & & $1.50 \mathrm{E}+01$ & 0.967 & 7.18 & 270 & 27 & 37.71 \\
\hline 56 & & & & & & & & & & & 0.979 & 2 & & & 77.47 \\
\hline 57 & & & & & & & $-1.27 \mathrm{E}-\mathrm{C}$ & & & & 0.983 & & & & 39.92 \\
\hline 58 & & & & & -1.1 & $E+01$ & & & 05 & -1.2 & 0.983 & 7.72 & & 15 & 04.21 \\
\hline 59 & 7 & +02 & $2.82 \mathrm{E}+00$ & +02 & $-1.23 \mathrm{E}-01$ & $2.92 \mathrm{E}+01$ & $-1.46 \mathrm{E}-01$ & $E+01$ & E-04 & $2.62 \mathrm{E}+01$ & 0.987 & 15.22 & 273 & 193.77 & 849.02 \\
\hline 60 & & & & & & & & & & $2.16 \mathrm{E}+01$ & 0.975 & 9.80 & 284 & 2.59 & 02.08 \\
\hline 1 & & & & & & & & & & & 0.988 & & 273 & & 298.99 \\
\hline 02 & & & & & & & & & & $\ldots$ & 0.978 & 18.75 & & & .53 \\
\hline 63 & & & -6.4 & & $-3.80 \mathrm{E}$ & $4.74 \mathrm{E}+01$ & $-2.00 \mathrm{E}-02$ & $\cdots$ & -05 & $-2.07 \mathrm{E}+01$ & 0.964 & 9.44 & 276 & 102.89 & 359.97 \\
\hline 64 & & & & $E+01$ & & $\ldots$ & & $23 \mathrm{E}+00$ & $-8.53 \mathrm{E}-06$ & $\ldots$ & 0.870 & 4.85 & 277 & 12.81 & 77.37 \\
\hline 65 & & & & & & & & & & & & & & & 5.93 \\
\hline $6(-2+2)$ & & & & & & & & $3.11 \mathrm{E}+1$ & & & 969 & 72 & 31 & & 1.88 \\
\hline 67 & & & & & 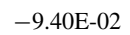 & & $-9.95 \mathrm{E}-\mathrm{C}$ & & & & & & 77 & 54 & 52.50 \\
\hline 68 & $7.73 \mathrm{E}+03$ & $8.66 \mathrm{E}+02$ & $-2.91 \mathrm{E}+00$ & $8.55 \mathrm{E}+02$ & $-1.54 \mathrm{E}-01$ & $7.85 \mathrm{E}+01$ & $-1.37 \mathrm{E}-01$ & $7.14 \mathrm{E}+01$ & $2.76 \mathrm{E}-04$ & & 0.984 & 17.69 & 277 & 177.46 & 845.72 \\
\hline 69 & & & $6.29 \mathrm{E}-01$ & $-1.94 \mathrm{E}+02$ & $-2.18 \mathrm{E}$ & & 2E-02 & $\mathrm{E}+01$ & -05 & & 0.984 & 8.41 & 265 & 49.75 & 19.83 \\
\hline 70 & & & & & $9.39 \mathrm{E}-0$ & & & & & & .991 & 9.61 & 269 & & 5.89 \\
\hline r & & & & & $\ldots$ & & & & & . & & 9.27 & 269 & & .39 \\
\hline 72 & $3 E+02$ & $\mathrm{E}+01$ & $-1.99 \mathrm{E}-01$ & $\mathrm{E}+01$ & $\ldots$ & $1.21 \mathrm{E}+01$ & $-1.33 \mathrm{E}-02$ & $5 \mathrm{E}+01$ & $1.74 \mathrm{E}-05$ & $\ldots$ & 0.976 & 5.31 & 274 & 47.53 & 236.68 \\
\hline 73 & $2.68 \mathrm{E}+03$ & $5.54 \mathrm{E}+02$ & $-8.35 \mathrm{E}-01$ & $6.33 \mathrm{E}+01$ & $-7.21 \mathrm{E}-02$ & $\cdots$ & $-1.09 \mathrm{E}-02$ & $6.26 \mathrm{E}+01$ & $6.71 \mathrm{E}-05$ & $\cdots$ & 0.987 & 5.89 & 270 & 20.75 & 300.08 \\
\hline
\end{tabular}




\begin{tabular}{|c|c|c|c|c|c|c|c|c|c|c|c|c|c|c|c|}
\hline \multicolumn{16}{|c|}{$\begin{array}{c}\text { Table } 4 \\
\text { (Continued) }\end{array}$} \\
\hline Index & $\bar{c}_{c_{0}}$ & $c_{1}$ & $c_{2}$ & $c_{3}$ & $c_{4}$ & $c_{5}$ & $c_{6}$ & $\bar{c}_{7}$ & $c_{8}$ & $\bar{c}_{c_{9}}$ & $\frac{\bar{R} R^{2}}{}$ & $\begin{array}{c}\sigma \\
(\mathrm{m} \AA)\end{array}$ & $\mathrm{N}$ & $\begin{array}{c}\mathrm{EW}_{\min } \\
(\mathrm{mA})\end{array}$ & $\begin{array}{c}\mathrm{EW}_{\max } \\
(\mathrm{m} \AA)\end{array}$ \\
\hline 74 & $1.19 \mathrm{E}+03$ & $1.13 \mathrm{E}+02$ & $-4.96 \mathrm{E}-01$ & $4.82 \mathrm{E}+02$ & $-1.03 \mathrm{E}-02$ & $4.23 \mathrm{E}+01$ & $-7.88 \mathrm{E}-02$ & $3.26 \mathrm{E}+01$ & 5.82E-05 & $\ldots$ & 0.990 & 8.03 & 276 & 202.99 & 604.88 \\
\hline 75 & $1.24 \mathrm{E}+03$ & $2.90 \mathrm{E}+02$ & $-1.33 \mathrm{E}-01$ & $-2.23 \mathrm{E}+02$ & $-1.82 \mathrm{E}-02$ & $-1.47 \mathrm{E}+01$ & $1.99 \mathrm{E}-02$ & $4.56 \mathrm{E}+01$ & $-3.53 \mathrm{E}-06$ & $1.16 \mathrm{E}+01$ & 0.982 & 5.58 & 280 & 17.49 & 237.20 \\
\hline 76 & $2.32 \mathrm{E}+03$ & $5.40 \mathrm{E}+02$ & $-6.80 \mathrm{E}-01$ & $7.71 \mathrm{E}+01$ & $-4.92 \mathrm{E}-02$ & $-1.17 \mathrm{E}+01$ & $-1.32 \mathrm{E}-02$ & $7.14 \mathrm{E}+01$ & $5.41 \mathrm{E}-05$ & $\cdots$ & 0.990 & 6.64 & 282 & 57.41 & 405.60 \\
\hline 77 & $2.91 \mathrm{E}+03$ & $3.00 \mathrm{E}+02$ & $-9.33 \mathrm{E}-01$ & $8.25 \mathrm{E}+01$ & $-2.74 \mathrm{E}-02$ & $-9.92 \mathrm{E}+00$ & $-5.32 \mathrm{E}-02$ & $1.81 \mathrm{E}+01$ & $9.31 \mathrm{E}-05$ & $2.71 \mathrm{E}+01$ & 0.983 & 5.63 & 271 & 80.82 & 346.14 \\
\hline 78 & $1.88 \mathrm{E}+03$ & $3.87 \mathrm{E}+02$ & $-6.33 \mathrm{E}-01$ & $1.53 \mathrm{E}+02$ & $-3.50 \mathrm{E}-02$ & $-1.61 \mathrm{E}+01$ & $-4.72 \mathrm{E}-02$ & $3.82 \mathrm{E}+01$ & $6.64 \mathrm{E}-05$ & $1.30 \mathrm{E}+01$ & 0.972 & 6.41 & 279 & 87.22 & 317.56 \\
\hline 79 & $4.64 \mathrm{E}+03$ & $6.55 \mathrm{E}+02$ & $-1.63 \mathrm{E}+00$ & $3.08 \mathrm{E}+02$ & $-7.55 \mathrm{E}-02$ & $-1.35 \mathrm{E}+01$ & $-5.24 \mathrm{E}-02$ & $3.51 \mathrm{E}+01$ & $1.48 \mathrm{E}-04$ & $\ldots$ & 0.983 & 8.92 & 274 & 90.97 & 506.80 \\
\hline 80 & $2.28 \mathrm{E}+03$ & $6.35 \mathrm{E}+02$ & $-6.00 \mathrm{E}-01$ & $-9.05 \mathrm{E}+01$ & $-6.72 \mathrm{E}-02$ & $-2.44 \mathrm{E}+01$ & $1.44 \mathrm{E}-02$ & $6.82 \mathrm{E}+01$ & $3.91 \mathrm{E}-05$ & $\ldots$ & 0.984 & 5.91 & 274 & 4.81 & 262.53 \\
\hline 81 & $-1.78 \mathrm{E}+01$ & $1.25 \mathrm{E}+01$ & 4.93E-02 & $-3.53 \mathrm{E}+00$ & $5.45 \mathrm{E}-03$ & $\cdots$ & $\cdots$ & $\cdots$ & $-6.20 \mathrm{E}-06$ & $\cdots$ & 0.944 & 2.97 & 275 & 10.24 & 73.26 \\
\hline 82 & $2.33 \mathrm{E}+02$ & $1.61 \mathrm{E}+02$ & $-3.68 \mathrm{E}-02$ & $3.14 \mathrm{E}+01$ & $\cdots$ & $-1.74 \mathrm{E}+01$ & $-7.89 \mathrm{E}-03$ & $2.53 \mathrm{E}+01$ & $3.65 \mathrm{E}-06$ & $\cdots$ & 0.973 & 3.82 & 273 & 23.46 & 145.70 \\
\hline 83 & $1.68 \mathrm{E}+03$ & $5.67 \mathrm{E}+02$ & $-4.07 \mathrm{E}-01$ & $-8.50 \mathrm{E}+01$ & $-5.96 \mathrm{E}-02$ & $-2.29 \mathrm{E}+01$ & $1.31 \mathrm{E}-02$ & $5.50 \mathrm{E}+01$ & $2.38 \mathrm{E}-05$ & $\cdots$ & 0.958 & 8.34 & 282 & 17.03 & 237.91 \\
\hline 84 & $4.80 \mathrm{E}+02$ & $3.10 \mathrm{E}+02$ & $-2.64 \mathrm{E}-02$ & $-3.56 \mathrm{E}+01$ & $-9.49 \mathrm{E}-03$ & $-2.56 \mathrm{E}+01$ & $\cdots$ & $2.61 \mathrm{E}+01$ & $\cdots$ & $\cdots$ & 0.969 & 7.01 & 287 & 70.26 & 272.69 \\
\hline 85 & $9.04 \mathrm{E}+02$ & $3.19 \mathrm{E}+02$ & $-3.12 \mathrm{E}-01$ & $1.80 \mathrm{E}+02$ & $\cdots$ & $-4.04 \mathrm{E}+01$ & $\cdots$ & $1.73 \mathrm{E}+01$ & $2.16 \mathrm{E}-05$ & $-2.32 \mathrm{E}+01$ & 0.943 & 10.38 & 289 & 50.34 & 288.82 \\
\hline 86 & $1.60 \mathrm{E}+03$ & $4.26 \mathrm{E}+02$ & $-4.31 \mathrm{E}-01$ & $-2.13 \mathrm{E}+01$ & $-2.43 \mathrm{E}-02$ & $-3.37 \mathrm{E}+01$ & $\cdots$ & $5.65 \mathrm{E}+01$ & $3.27 \mathrm{E}-05$ & $\cdots$ & 0.949 & 9.23 & 288 & 27.81 & 269.08 \\
\hline 87 & $8.09 \mathrm{E}+02$ & $4.29 \mathrm{E}+02$ & $-1.23 \mathrm{E}-01$ & $5.13 \mathrm{E}+00$ & $-2.48 \mathrm{E}-02$ & $-2.56 \mathrm{E}+01$ & $2.35 \mathrm{E}-02$ & $5.00 \mathrm{E}+01$ & $-5.56 \mathrm{E}-06$ & $-1.81 \mathrm{E}+01$ & 0.977 & 7.58 & 279 & 59.74 & 312.23 \\
\hline 88 & $2.96 \mathrm{E}+02$ & $1.37 \mathrm{E}+02$ & $-1.74 \mathrm{E}-03$ & $-6.35 \mathrm{E}+01$ & $\ldots$ & $-1.56 \mathrm{E}+01$ & $9.61 \mathrm{E}-03$ & $1.55 \mathrm{E}+01$ & $-5.55 \mathrm{E}-06$ & $\ldots$ & 0.956 & 4.04 & 290 & 13.13 & 117.75 \\
\hline 89 & $1.43 \mathrm{E}+03$ & $3.10 \mathrm{E}+02$ & $-2.88 \mathrm{E}-01$ & $-6.82 \mathrm{E}+01$ & $-1.04 \mathrm{E}-02$ & $-1.97 \mathrm{E}+01$ & $1.02 \mathrm{E}-02$ & $2.95 \mathrm{E}+01$ & $1.36 \mathrm{E}-05$ & $\cdots$ & 0.986 & 5.70 & 275 & 62.96 & 297.99 \\
\hline 90 & $1.17 \mathrm{E}+03$ & $3.31 \mathrm{E}+02$ & $-3.68 \mathrm{E}-01$ & $1.11 \mathrm{E}+02$ & $-3.58 \mathrm{E}-02$ & $\cdots$ & $-1.81 \mathrm{E}-02$ & $3.37 \mathrm{E}+01$ & $3.18 \mathrm{E}-05$ & $\cdots$ & 0.989 & 4.28 & 275 & 48.27 & 262.34 \\
\hline 91 & $9.60 \mathrm{E}+02$ & $1.77 \mathrm{E}+02$ & $-2.73 \mathrm{E}-01$ & $4.37 \mathrm{E}+01$ & $-6.48 \mathrm{E}-03$ & $-1.01 \mathrm{E}+01$ & $\cdots$ & $2.81 \mathrm{E}+01$ & $1.90 \mathrm{E}-05$ & $-6.29 \mathrm{E}+00$ & 0.989 & 3.00 & 266 & 22.05 & 157.34 \\
\hline 92 & $1.40 \mathrm{E}+03$ & $3.18 \mathrm{E}+02$ & $-6.53 \mathrm{E}-01$ & $4.47 \mathrm{E}+02$ & $-6.81 \mathrm{E}-02$ & $4.72 \mathrm{E}+01$ & $-5.77 \mathrm{E}-02$ & $1.23 \mathrm{E}+01$ & $6.69 \mathrm{E}-05$ & $-8.61 \mathrm{E}+00$ & 0.991 & 4.93 & 263 & 83.85 & 347.46 \\
\hline 93 & $1.85 \mathrm{E}+03$ & $3.10 \mathrm{E}+02$ & $-8.84 \mathrm{E}-01$ & $6.41 \mathrm{E}+02$ & $-6.29 \mathrm{E}-02$ & $5.64 \mathrm{E}+01$ & $-5.19 \mathrm{E}-02$ & $9.02 \mathrm{E}+00$ & 8.04E-05 & $-3.39 \mathrm{E}+01$ & 0.990 & 7.30 & 271 & 113.74 & 474.63 \\
\hline 94 & $1.11 \mathrm{E}+03$ & $2.05 \mathrm{E}+02$ & $-5.11 \mathrm{E}-01$ & $3.19 \mathrm{E}+02$ & $-3.74 \mathrm{E}-02$ & $2.15 \mathrm{E}+01$ & $-3.49 \mathrm{E}-02$ & $3.73 \mathrm{E}+00$ & $5.06 \mathrm{E}-05$ & $-1.17 \mathrm{E}+01$ & 0.986 & 3.77 & 265 & 62.06 & 265.81 \\
\hline 95 & $4.04 \mathrm{E}+02$ & $1.00 \mathrm{E}+02$ & $4.59 \mathrm{E}-02$ & $-1.45 \mathrm{E}+02$ & $1.62 \mathrm{E}-02$ & $-2.85 \mathrm{E}+01$ & $2.16 \mathrm{E}-02$ & $2.72 \mathrm{E}+01$ & $-1.56 \mathrm{E}-05$ & $\cdots$ & 0.956 & 4.74 & 270 & 12.75 & 129.88 \\
\hline 96 & $1.36 \mathrm{E}+03$ & $-5.09 \mathrm{E}+01$ & $-9.19 \mathrm{E}-01$ & $9.02 \mathrm{E}+02$ & $-2.51 \mathrm{E}-02$ & $8.31 \mathrm{E}+01$ & $-9.26 \mathrm{E}-02$ & $-2.45 \mathrm{E}+01$ & $9.79 \mathrm{E}-05$ & $-3.40 \mathrm{E}+01$ & 0.988 & 8.72 & 266 & 113.80 & 514.74 \\
\hline
\end{tabular}

precise atmospheric parameters $\left(T_{\text {eff }},[\mathrm{Fe} / \mathrm{H}]\right.$, and $\left.\log g\right)$ derived from detailed and homogeneous analysis of high-resolution spectra. With both the EWs and atmospheric parameters in hand, we explored the relations between these quantities through a multivariate analysis and the final result was a set of calibrations that allowed the subsequent characterization of the MARVELS validation sample based solely on spectral indices (without any other priors).

Figure 5 shows an example of the behavior of the indices' EWs as a function the atmospheric parameters for the calibration sample. Clear relations are readily visible for $T_{\text {eff }}$ and $[\mathrm{Fe} / \mathrm{H}]$, while only scatter can be seen for $\log g$. Although this initial qualitative analysis revealed some promising indices, it can be misleading for the cases which lack evident correlations. The scatter might result from a truly weak sensitivity of the index relative to the parameter in question, but can also be caused by a stronger sensitivity to one of the other two parameters (recall that these plots show only a 1D projection of a possibly threedimensional (3D) relation). Problems during the measurement of the EWs for particular indices (e.g., possible contaminations having larger contributions than anticipated) certainly also play a role. Therefore, we decided not to remove any of the indices based on this initial visual inspection.

The starting point of the quantitative analysis was the choice of an appropriate model to describe the relations observed in plots similar to Figure 5. In principle, all indices' EWs depend on the three atmospheric parameters in a complicated way which includes the interdependency between these parameters. Tests with different polynomial orders revealed that a quadratic model would be able to correctly describe most of the observed behaviors. Thus, we decided to search for the best calibrations for each index using second-order polynomials with the following structure.

$$
\begin{aligned}
\mathrm{EW}(\mathrm{m \AA})= & c_{0}+c_{1}[\mathrm{Fe} / \mathrm{H}]+c_{2} T_{\text {eff }}+c_{3} \log g \\
& +c_{4}[\mathrm{Fe} / \mathrm{H}] T_{\text {eff }}+c_{5}[\mathrm{Fe} / \mathrm{H}] \log g+c_{6} T_{\text {eff }} \log g \\
& +c_{7}([\mathrm{Fe} / \mathrm{H}])^{2}+c_{8}\left(T_{\text {eff }}\right)^{2}+c_{9}(\log g)^{2} .
\end{aligned}
$$

These models ensure that any eventual interdependence between the atmospheric parameters are taken into account. Some of the terms, however, could be statistically insignificant and, to evaluate this possibility, the choice of the final best calibrations followed an iterative procedure, which was performed with a Python routine developed for this work. The starting point was Equation (1), for which the coefficients $c_{0}, \ldots, c_{9}$ were determined through the ordinary least squares method (i.e., without any weights). We have designated these the complete models. When the best fit was found, the outliers were removed with a $2 \sigma$ clipping. The previous steps were repeated three times and this limit was chosen to avoid an excessive exclusion of points. The mean number of stars that appeared as outliers was 37, with minimum and maximum values of 19 and 49 , respectively. These numbers correspond to $\sim 6 \%-16 \%$ of the complete calibration sample, which is a reasonable fraction. We have checked that these outliers do not correspond to the stars that had their normalizations replaced by visually better fits (see Section 4.1). At the end of this iteration, the following statistical quantities were obtained for the current model: the correlation coefficient $R^{2}$ of the fit, the standard deviation of the residuals $(\sigma)$ and the Bayesian Information Criterion (BIC).

The code then searched for models that had the same polynomial order and a similar ability to fit the data, but with fewer terms; these are the reduced models. The progression of these tests was hierarchical, i.e., the higher-order terms were the first ones to be removed. In each trial, a temporary reduced model and its associated statistical quantities $\left(R^{2}\right.$, $\sigma$ and BIC) were calculated. This reduced model was then compared to the complete one and the temporarily removed term was definitely excluded from the polynomial if three criteria were simultaneously met: both $\sigma$ and BIC are lower for the reduced model and the corresponding coefficient $c_{i}$ is statistically insignificant according to the $F$ statistics. If these conditions were satisfied, the reduced model replaced the complete one and new trials were done until all terms were tested. If one of the conditions was not satisfied, the tested term returned to the model and the trials continued until 


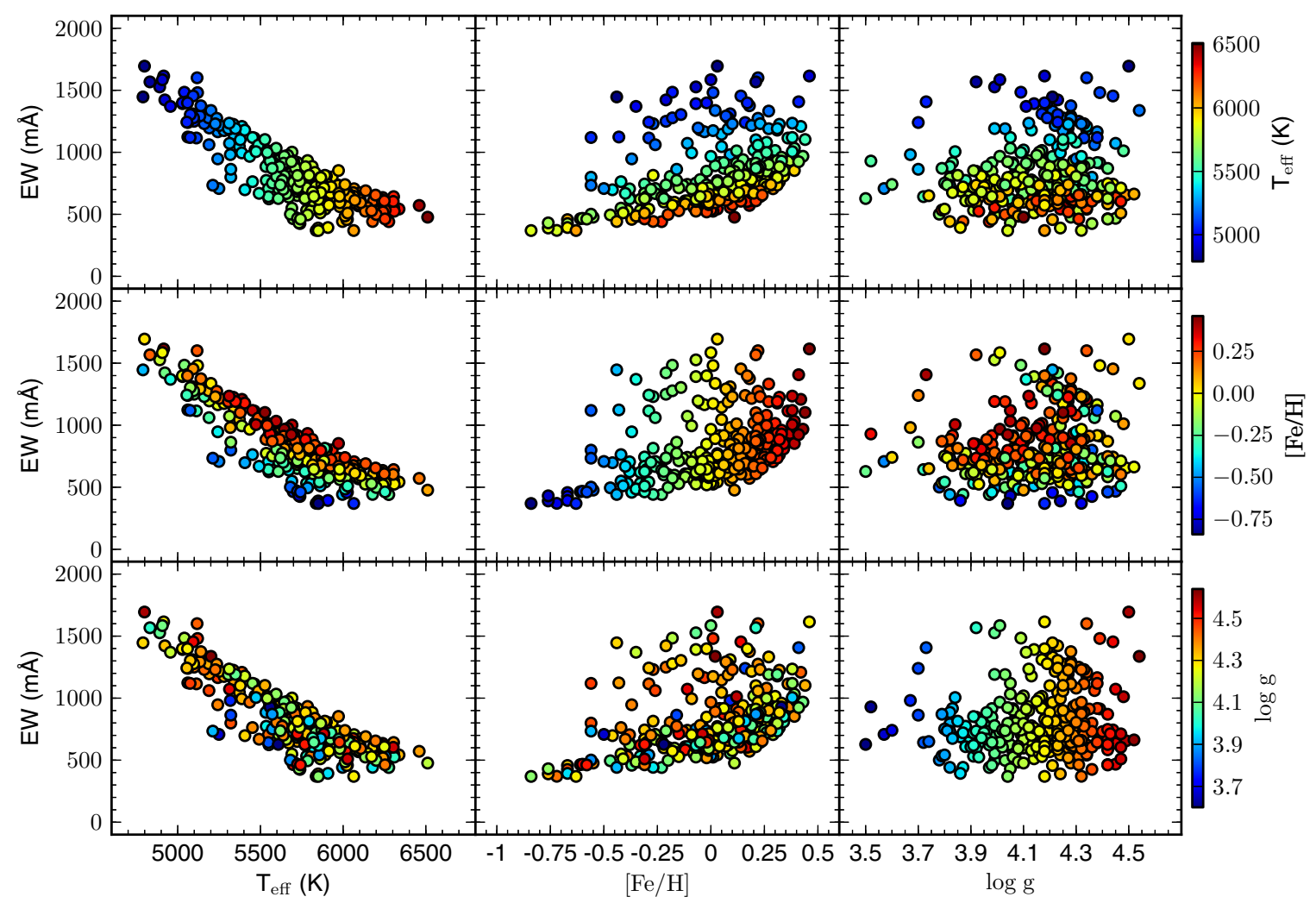

Figure 5. Measured equivalent widths of index 38 (see Table 3) as a function of the atmospheric parameters. The left, middle, and right columns show the dependencies with $T_{\text {eff }},[\mathrm{Fe} / \mathrm{H}]$, and $\log g$, respectively. In the upper, middle, and lower rows, the points are color-coded according to their effective temperatures, metallicities, and surface gravities, respectively. The EWs exhibit significant correlations with $T_{\text {eff }}$ and $[\mathrm{Fe} / \mathrm{H}]$, but no visible dependency on $\log g$ is observed.

(A color version and supplemental data of this figure are available in the online journal.)

the relevance of all terms was evaluated. After the iterative procedure described above, a final optimized calibration was obtained for each index.

Table 4 presents the information for the calibrations obtained for the total set of 96 indices. If no number is provided for a given coefficient, the corresponding term was excluded according to the tests of statistical significance explained above. Besides $R^{2}$ and $\sigma$, the number of stars used in the fit and the validity ranges for the EWs are also shown. The validity ranges for $T_{\text {eff }}$, $[\mathrm{Fe} / \mathrm{H}]$, and $\log g$ are not given because they are very similar to the parameter space covered by the calibration sample (see Section 2.1).

In order to guarantee the determination of precise atmospheric parameters, we have decided to keep in the analysis only those indices for which the respective calibrations had $R^{2} \geqslant 0.9$. This cut removed four indices (marked with a number 2 in the notes of Table 3): 1, 41, 64, and 65. Figure 6 shows index 38 as an example of the final calibrations adopted in this work.

The next step was the analysis of the residuals (i.e., the differences $\Delta \mathrm{EW}$ between the equivalent widths $\mathrm{EW}_{\text {calc }}$ calculated with Equation (1) and the equivalent widths $\mathrm{EW}_{\text {obs }}$ measured in the stellar spectra) for the remaining 92 calibrations. These were plotted as a function of the variables $\left(\mathrm{EW}, T_{\mathrm{eff}},[\mathrm{Fe} / \mathrm{H}]\right.$, and $\log g$ ) and linear fits were applied to the data to evaluate the existence of any systematics. An example is presented in Figure 7 for the same index as in Figures 5 and 6. The average correlation coefficient $R^{2}$ for the fits in which $\mathrm{EW}_{\mathrm{obs}}$ is the independent variable is $0.024 \pm 0.017$, with minimum and maximum values 0.006 and 0.096 , respectively. For the cases with $T_{\text {eff }},[\mathrm{Fe} / \mathrm{H}]$, and $\log g$ as the independent variables, the values of $R^{2}$ are essentially null for all indices. These results demonstrate that the residuals are free of any significant systematic trends and there is no need to apply any a posteriori linear corrections to the EWs calculated with Equation (1).

\subsection{Determination of the Atmospheric Parameters}

The last of the three codes developed for this work delivers the final products of our analysis, which are the atmospheric parameters and their associated uncertainties. To accomplish this task, the program only requires the EWs and calibrations discussed in Sections 4.2 and 4.3, respectively. These can be changed in order to account for differences in the resolution and wavelength range of the spectra. The choice of the best atmospheric parameters for each star is based on the minimization of a reduced chi-square $\left(\chi_{r}^{2}\right)$ and is described below.

First, a set of 92 theoretical EWs (one for each index) is calculated for each point of a $3 \mathrm{D}$ grid of atmospheric parameters. The grid covers the following intervals: $4700 \mathrm{~K} \leqslant T_{\text {eff }} \leqslant 6600 \mathrm{~K}$, with $10 \mathrm{~K}$ steps; $-0.90 \leqslant[\mathrm{Fe} / \mathrm{H}] \leqslant 0.50$, with 0.02 dex steps; and $3.50 \leqslant \log g \leqslant 4.70$, with 0.05 dex steps. These ranges are consistent with the parameter space covered by the calibration sample. Smaller steps were tested, but no improvements in the results were observed. Then, for each set of atmospheric parameters, a comparison between the observed and theoretical EWs is performed through the calculation of a reduced chisquare as follows:

$$
\chi_{r}^{2}=\frac{1}{N_{\text {ind }}} \sum_{i=1}^{N_{\text {ind }}} \frac{\left(\mathrm{EW}_{i}^{\mathrm{obs}}-\mathrm{EW}_{i}^{\mathrm{calc}}\right)^{2}}{\sigma_{i}^{2}} .
$$



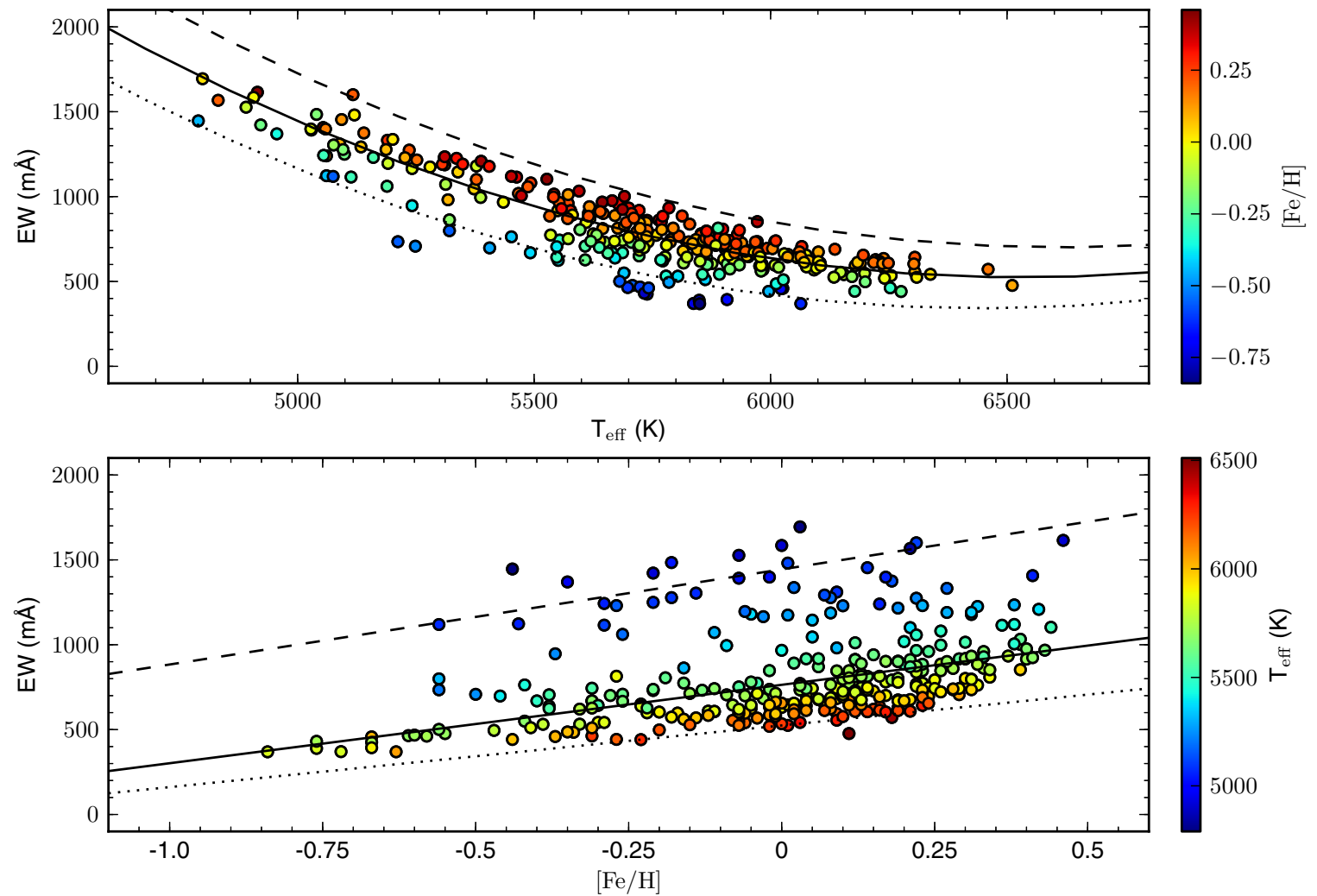

Figure 6. Examples of the calibration for index 38 (see Table 4). Upper panel: Variation of the measured equivalent widths as a function of $T_{\text {eff }}$, with the points color-coded by $[\mathrm{Fe} / \mathrm{H}]$. The dotted, solid, and dashed black lines represent Equation (1) for $\log g=4.44$ and $[\mathrm{Fe} / \mathrm{H}]=-0.50,0.00$, and +0.50 , respectively. Lower panel: Variation of the measured equivalent widths as a function of $[\mathrm{Fe} / \mathrm{H}]$, with the points color-coded by $T_{\text {eff }}$. The dashed, solid, and dotted black lines represent Equation (1) for $\log g=4.44$ and $T_{\text {eff }}=5000,5750$, and $6500 \mathrm{~K}$, respectively. The calibration accurately represents the observed behavior of the points throughout the parameter space covered by the calibration sample.

(A color version and supplemental data of this figure are available in the online journal.)

In the above equation, $\mathrm{EW}_{i}^{\mathrm{obs}}$ and $\mathrm{EW}_{i}^{\text {calc }}$ are the EWs of the $i$ th index measured on the observed spectra and calculated with the calibrations, respectively. The number of indices used is given by $N_{\text {ind }}$. Finally, $\sigma_{i}$ is an error associated with the EWs. For the calibration sample, it is simply the standard deviation found for each calibration, i.e., $\sigma_{i}=\sigma_{i}^{\text {calib }}$. For the MARVELS validation sample, $\sigma_{i}$ is given by the quadratic sum of this term and the standard deviation of the average EWs measured on the stellar spectra, i.e., $\sigma_{i}=\sqrt{\left(\sigma_{i}^{\text {calib }}\right)^{2}+\left(\sigma_{i}^{\text {star }}\right)^{2}}$ (more details in Section 5.2).

The final atmospheric parameters are those that produce the minimum value of $\chi_{r}^{2}$. This value is also used for the determination of the associated uncertainties. First, we consider all sets of parameters that have $\left(\chi_{r}^{2}\right)_{\min } \leqslant \chi_{r}^{2} \leqslant 2\left(\chi_{r}^{2}\right)_{\min }$, then calculate the differences between these parameters and those considered to be the best ones (corresponding to $\left(\chi_{r}^{2}\right)_{\min }$ ). Finally, the root mean squares of these differences are taken as the errors of the parameters.

\section{RESULTS AND DISCUSSION}

The previous sections were devoted to a description of the method that was used to derive the atmospheric parameters based only on the EWs of spectral indices. We now discuss the tests performed to show that this approach is capable of recovering the precise atmospheric parameters determined from high-resolution stellar spectra and model atmosphere analysis.
Table 5

Comparison Between Atmospheric Parameters from Spectral Indices and High-resolution Analyses

\begin{tabular}{lcccc}
\hline \hline Sample & $\begin{array}{c}\Delta T_{\text {eff }} \\
(\mathrm{K})\end{array}$ & $\Delta[\mathrm{Fe} / \mathrm{H}]$ & $\Delta \log g$ & $\begin{array}{c}\text { Number of } \\
\text { Stars/Spectra }\end{array}$ \\
\hline \multicolumn{5}{c}{ Complete Set of 92 Indices } \\
\hline Calibration & $1 \pm 78$ & $0.01 \pm 0.06$ & $0.00 \pm 0.15$ & 309 \\
MARVELS & $-97 \pm 110$ & $0.00 \pm 0.07$ & $-0.30 \pm 0.17$ & 30 \\
ELODIE & $15 \pm 125$ & $0.06 \pm 0.10$ & $0.07 \pm 0.29$ & 219 \\
\hline \multicolumn{5}{c}{ Restricted Set of 64 Indices } \\
\hline Calibration & $-3 \pm 80$ & $0.01 \pm 0.06$ & $0.00 \pm 0.15$ & 309 \\
MARVELS & $-28 \pm 81$ & $0.02 \pm 0.05$ & $-0.07 \pm 0.15$ & 30 \\
\hline
\end{tabular}

\subsection{Application of the Method to the Calibration Sample}

The first test was a sanity check with the calibration sample. The same EWs employed to build the calibrations were used to derive the atmospheric parameters for the 309 calibration stars. The comparison between these results and those derived from high-resolution analysis is presented in Figure 8. The average differences obtained for each parameter are given in the upper part of Table 5.

The offsets between the two sets of parameters are negligible and the dispersions around the average residuals are lower or of the order of the external errors usually found in high-resolution analyses (for instance, see Table 5 of Ghezzi et al. 2010b). The situation is similar for the internal uncertainties of the method (based on the $\chi_{r}^{2}$; see Section 4.4), which have typical values 

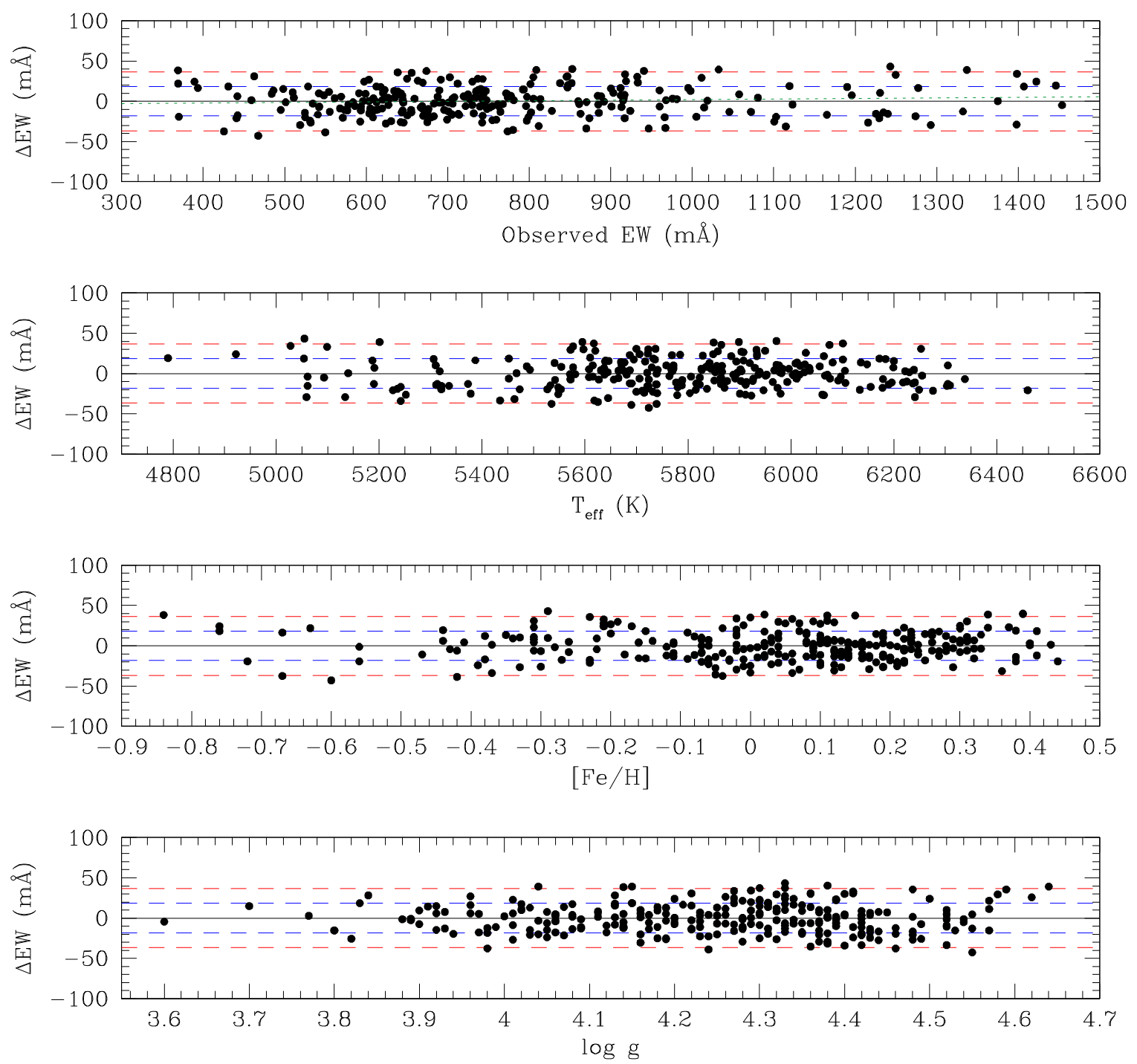

Figure 7. Residuals $\left(\Delta \mathrm{EW}=\mathrm{EW}_{\mathrm{calc}}-\mathrm{EW}_{\mathrm{obs}}\right)$ from the calibrations of index 38 as a function of, from top to bottom, $\mathrm{EW}_{\mathrm{obs}}, T_{\mathrm{eff}},[\mathrm{Fe} / \mathrm{H}]$, and log $g$. The horizontal black solid lines indicate the perfect agreement. The blue and red dashed lines show, respectively, the $1 \sigma$ and $2 \sigma$ limits, where $\sigma=18.36 \mathrm{~m} \AA$ is the standard deviation of the residuals (see Section 4). The green dotted line in the upper panel represents the linear fit to the residuals, which has a correlation coefficient $R^{2}=0.007$. In the panels for $T_{\text {eff }},[\mathrm{Fe} / \mathrm{H}]$, and $\log g$, the fit cannot be distinguished from the black line. No significant trends can be observed for the residuals as a function of the EWs and atmospheric parameters.

(A color version of this figure is available in the online journal.)

in the ranges $50-150 \mathrm{~K}$ for $T_{\text {eff }}, 0.05-0.10$ dex for $[\mathrm{Fe} / \mathrm{H}]$ and $0.10-0.25$ dex for $\log g$. The obvious outlier in the middle row panels of Figure 8 is a star that was excluded for 94 indices during the regressive analysis (see Section 4.3).

No significant trends can be seen in the residuals, except for systematically higher and lower $T_{\text {eff }}$ values at $\sim 5400 \mathrm{~K}$ and above $\sim 6300 \mathrm{~K}$, respectively. The latter behavior is probably caused by the reduced number of calibration stars (only seven) or by the fact that most of the indices become weaker in this temperature interval. This hypothesis is further supported by the behavior of the internal uncertainties; slight increases can be observed toward higher effective temperatures or lower metallicities. In both regimes, we have fewer stars and smaller EWs. The behavior at $\sim 5400 \mathrm{~K}$ could be related to the discontinuity in the number of calibrations stars with cooler and hotter effective temperatures (see Figure 1). This systematic effect, however, has a small amplitude, with the majority of the residuals being within $\pm 100 \mathrm{~K}$.
As a complementary check, we have also analyzed the FEROS solar spectrum from Ghezzi et al. (2010b) with the same approach as above. The derived atmospheric parameters were: $T_{\text {eff }}=5720 \pm 95 \mathrm{~K},[\mathrm{Fe} / \mathrm{H}]=-0.02 \pm 0.06$, and $\log g=$ $4.30 \pm 0.16$. We can see that there is a good agreement with the standard solar parameters within the uncertainties. This result is expected given that the selection of the indices was based on a Ganymede spectrum used as a solar template. The above results thus provide a confirmation that the spectral indices method produces internally consistent atmospheric parameters.

\subsection{Application of the Method to the MARVELS Validation Sample}

The second test had the goal of demonstrating that the method is capable of accurately recovering the high-resolution atmospheric parameters of a given stellar sample that was not used in the calibration of the method. To perform this exercise, 

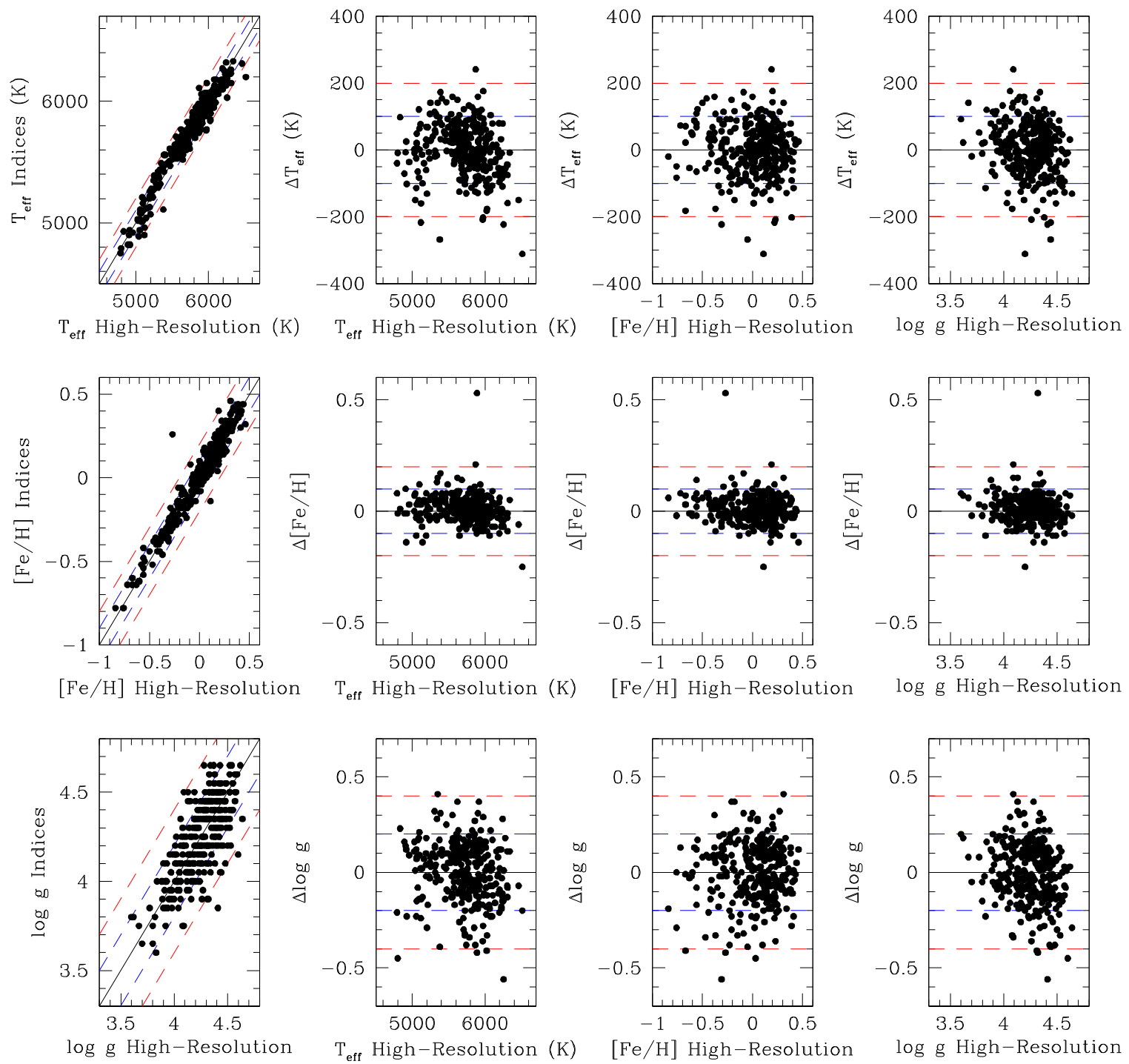

Figure 8. Comparison between atmospheric parameters derived from spectral indices and high-resolution analyses for the calibration sample. The first column shows the direct comparison between the two sets of parameters, with $T_{\text {eff }},[\mathrm{Fe} / \mathrm{H}]$, and $\log g$ on the top, middle, and bottom rows, respectively. The differences between the results from the indices and high-resolution as a function of the latter are presented in the remaining panels. The differences for $T_{\text {eff }}$, [Fe/H], and $\log g$ are shown in the top, middle, and bottom rows, respectively. The dependency with the high-resolution effective temperatures, metallicities, and surface gravities are given in columns 2,3 , and 4, respectively. In all panels, the solid black line represents perfect agreement. The dashed blue and red lines mark, respectively, the offsets of \pm 100 and $\pm 200 \mathrm{~K}$ for $T_{\text {eff }}, \pm 0.10$ and \pm 0.20 dex for $[\mathrm{Fe} / \mathrm{H}]$, and \pm 0.20 and \pm 0.40 dex for $\log g$. There is a good agreement between the two sets of atmospheric parameters and no significant offsets and trends in the residuals.

(A color version of this figure is available in the online journal.)

we used the validation sample of 30 stars observed as part of the MARVELS survey (see Section 2.2).

The approach described in Section 4 was used to obtain the atmospheric parameters from the reduced, defringed, 1D, Doppler-corrected MARVELS spectra. However, a few remarks are necessary. All MARVELS stars have two sets of spectra because their light is divided into two "beams" that are collected by two neighboring fibers (see Section 2.2). Each set of spectra was analyzed separately and the exposures within each group were not combined for the normalization. This procedure was done because we wanted to have multiple measurements for the EWs of the indices in order to remove possible outliers and to have an estimate of their uncertainties. The average EWs obtained after an iterative $2 \sigma$ clipping (until there were no outliers left) were used as input to the atmospheric parameter's determination method. The $\sigma$ used in the calculation of $\chi_{r}^{2}$ was given by the quadratic sum of $\sigma_{i}^{\text {calib }}$ and $\sigma_{i}^{\text {star }}$, as previously mentioned in Section 4.4. Finally, the two sets of parameters for each star were combined using a simple arithmetic average, with the uncertainties being obtained through an error propagation.

The results in this initial attempt were not acceptable. The offsets relative to the high-resolution parameters and the dispersions were too large (see the upper part of Table 5). Moreover, there were significant systematic trends as a function of $T_{\text {eff }}$. The only possible causes for these issues were the two types of input data used in our method: the measured EWs or the calibrations. However, the good results obtained from the test with the calibration sample ruled out the latter. Direct comparisons between the EWs measured in the FEROS degraded and MARVELS solar spectra revealed significant differences. However, these differences were not caused by problems in the normalization and did not present any systematic behavior as 
a function of $\lambda, T_{\text {eff }},[\mathrm{Fe} / \mathrm{H}], \log g$, and the EWs themselves. Therefore, we decided to derive individual corrections for each index to place the MARVELS EWs onto the FEROS scale.

\subsubsection{Restriction of the Set of Indices and Correction of the EW Scale}

In order to derive the corrections, we have considered 120 MARVELS solar spectra (one for each fiber of the instrument) observed simultaneously on 2009 November 17. There is a different wavelength solution for each fiber; thus, the ranges covered vary from one spectrum to another. To avoid having indices with significantly fewer EW measurements than others, we have decided to use only the wavelength range that is present in all 120 exposures, which corresponds to the interval 5137-5543 $\AA$. As a consequence of this choice, 18 indices were removed from our analysis: $2-7$ on the blue end (note that 1 was already excluded because its calibration had $R^{2}<0.9$ ) and $85-96$ on the red end (all marked with a number 3 in the notes of Table 3 ).

For the remaining 74 indices, the average EWs from the 120 solar spectra and their respective standard deviations were calculated. No clipping was performed because we wanted to retain all of the information regarding the variations of the EWs with the fiber number in the instrument. This is important because the MARVELS stars could have been observed with any of the 120 fibers. We then calculated the differences $\left|\left\langle\mathrm{EW}_{\text {MARVELS }}\right\rangle-\mathrm{EW}_{\mathrm{FEROS}}\right|$. If these differences were higher than the corresponding values for $\sigma\left(\left\langle\mathrm{EW}_{\mathrm{MARVELS}}\right\rangle\right)$, the indices were removed. This was the case for the following 10 indices (marked with a number 4 in the notes of Table 3): 33, 34, 39, 40, 54, 55, 61, 62, 66, and 67.

Finally, it was decided that the remaining 64 indices would have their average MARVELS EWs corrected by the difference $\left|\left\langle\mathrm{EW}_{\text {MARVELS }}\right\rangle-\mathrm{EW}_{\text {FEROS }}\right|$. Although this correction was only based on the Sun, it was applied to all stars in the validation sample, regardless of their parameters. As it is shown below, the correction proved to be effective. Another constraint implemented for the analysis of the MARVELS stars was the removal of corrected average EWs that were outside the ranges defined by each of the calibrations (see Table 4). This cut was done to avoid any extrapolation outside the parameter space defined by our calibration sample.

\subsubsection{Final Results}

Using the method with the above optimization to the MARVELS data, we rederived the atmospheric parameters for the 30 stars in the MARVELS validation sample. Recall that the final results for each object were obtained from an arithmetic average of the parameters produced by its two associated fibers. The two sets of results are in good agreement, with average differences of $-18 \pm 91 \mathrm{~K}$ for $T_{\text {eff }}, 0.01 \pm 0.06 \operatorname{dex}$ for $[\mathrm{Fe} / \mathrm{H}]$ and $-0.09 \pm 0.16 \mathrm{dex}$ for $\log g$. These values highlight that the data from adjacent fibers on the MARVELS instrument are consistent.

The comparison between the atmospheric parameters derived from the indices and high-resolution analyses is presented in Figure 9. The average differences obtained for each parameter are given in the lower part of Table 5 . The offset in the metallicity is once again close to zero. For $T_{\text {eff }}$, we have a non-negligible negative value, but it is lower than the dispersion of the residuals and the typical uncertainties found for this parameter. A similar situation was found for $\log g$. However, the offset in this case can be traced back to the reference values for the surface gravity. We recall that the values for the calibration sample present a small offset relative to other literature values (see Table 5 of Ghezzi et al. 2010b).

The dispersions around the average offsets are either lower than, or on the order of, the typical external uncertainties of high-resolution analyses. This was also the case for the internal uncertainties of the method, which have typical values in the ranges of $80-160 \mathrm{~K}$ for $T_{\text {eff }}, 0.05-0.10 \mathrm{dex}$ for $[\mathrm{Fe} / \mathrm{H}]$, and $0.15-0.25 \mathrm{dex}$ for $\log g$. All these values are close to the ones derived for the calibration sample. The residuals do not exhibit any significant trends, except possibly for the same ones observed for $T_{\text {eff }}$ in the results for the calibration sample.

The residuals and internal uncertainties also do not exhibit significant trends as a function of the $\mathrm{S} / \mathrm{N}$ values of the spectra or the $V$ magnitudes of the stars (taken from the Guide Star Catalog; Lasker et al. 2008), as can be seen on Figure 10. The total S/ $\mathrm{N}$ for a given star was obtained from the arithmetic average of the values derived from its two sets of spectra (one from each fiber). The $\mathrm{S} / \mathrm{N}$ for each set was calculated by multiplying the average $\mathrm{S} / \mathrm{N}$ of the individual spectra $\left\langle(\mathrm{S} / \mathrm{N})_{\mathrm{spec}}\right\rangle$ by the square root of the number of spectra $\sqrt{N_{\text {spec }}}$. This is a reasonable approximation considering that the exposure times are similar for all spectra of a given star.

The lack of a dependency between the accuracy and precision (differences and errors in the left and right columns of Figure 10, respectively) of the results and the $\mathrm{S} / \mathrm{N}$ of the spectra can be explained by a particular characteristic of our data. All stars have $\mathrm{S} / \mathrm{N} \geqslant 200 \mathrm{pixel}^{-1}$ (dispersion $0.154 \AA \mathrm{pixel}^{-1}$ ) and this value is certainly higher than the limit below which we would start to observe a decline in the quality of the results with decreasing $\mathrm{S} / \mathrm{N}$. Thus, we conclude that all stars have high-quality data that allow our approach to work at its best precision and accuracy.

The method was further tested using the 120 MARVELS solar spectra previously described. Since we wanted to obtain the best possible results, an iterative $2 \sigma$ clipping (until there were no outliers left) was applied to calculate the average EWs of the Sun. Following the exact same method employed for the MARVELS validation sample, we derived the results: $T_{\text {eff }}=5760 \pm 81 \mathrm{~K},[\mathrm{Fe} / \mathrm{H}]=-0.04 \pm 0.05$ and $\log g=$ $4.40 \pm 0.15$. The agreement with the standard solar parameters is excellent.

For completeness, we also utilized the restricted set of 64 indices (without any corrections) to rederive the parameters for the calibration stars. As can be seen on the lower part of Table 5, there are no significant differences relative to the previous case in which all 92 indices were considered.

The above results for the MARVELS validation sample show that the spectral indices approach is able to accurately recover the precise atmospheric parameters derived from the analysis of high-resolution spectra. Moreover, the differences between the two sets of results and the internal uncertainties of the method presented here are both consistent with the typical external errors obtained in high-resolution analyses. It should be highlighted that this is achieved by using only EWs directly measured on the observed low-resolution MARVELS spectra, with no other priors.

\subsection{Application of the Method to the ELODIE Stellar Library}

The third and last test was conducted to complement the previous one. Although the validation sample contains stars with real spectra from the MARVELS survey, we recognize that 30 stars is a relatively small number to critically evaluate the performance of our method. Therefore, we decided to analyze the spectra contained in the ELODIE stellar library 

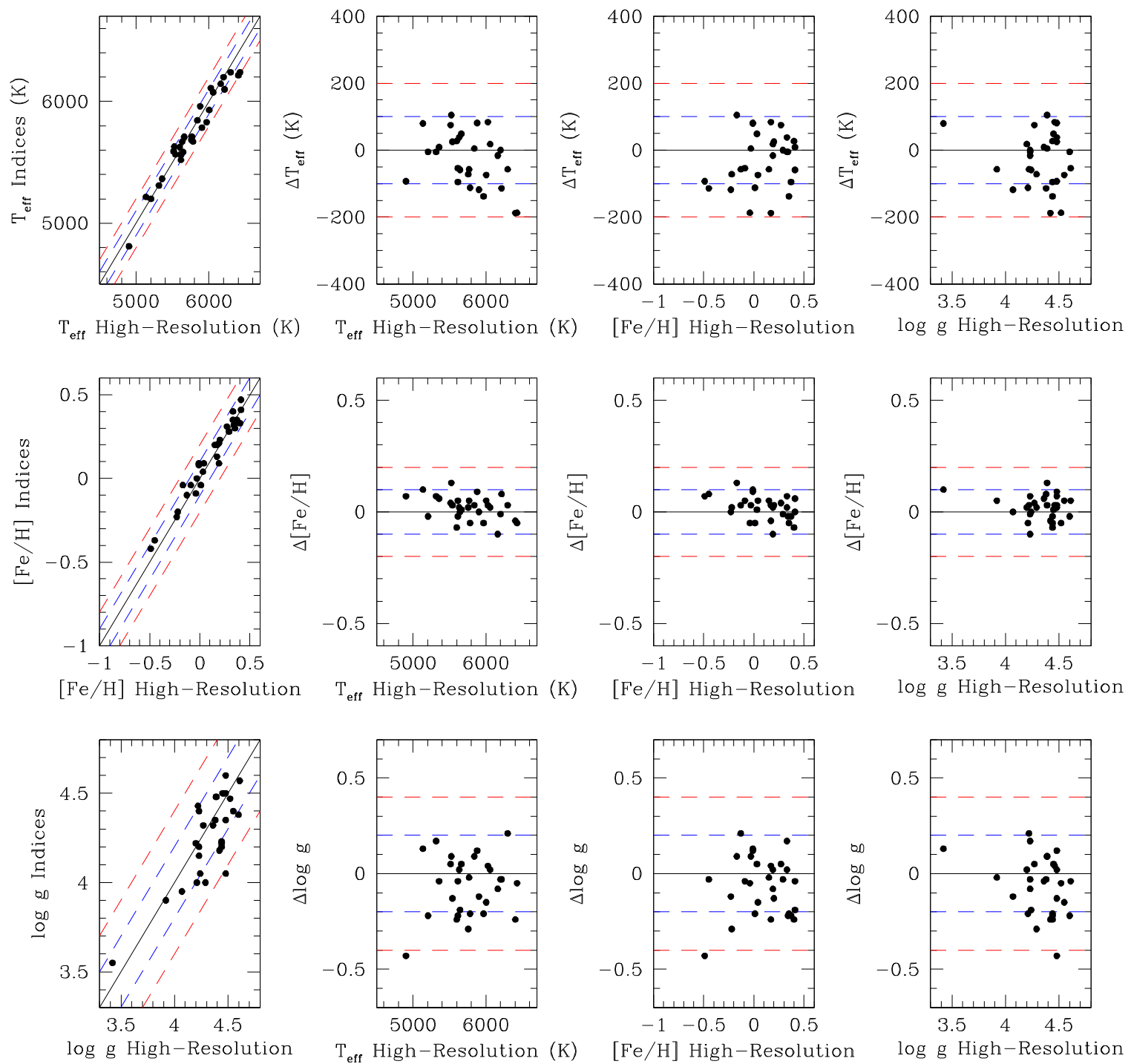

Figure 9. Comparison between atmospheric parameters derived from spectral indices and high-resolution analyses for the MARVELS validation sample. The panels, symbols, and lines have the same meaning as in Figure 8. A good agreement between the two sets of atmospheric parameters can be observed and there are no clear offsets and trends in the residuals.

(A color version of this figure is available in the online journal.)

(Prugniel \& Soubiran 2001; Prugniel et al. 2007; see Web site $^{23}$ for the most updated 3.1 version). Briefly, the library includes 1962 high-resolution $(R=42,000)$ spectra observed with ELODIE spectrograph coupled to the $1.93 \mathrm{~m}$ telescope at Observatoire de Haute-Provence. The spectra cover the wavelength range $3900-6800 \AA$, have $\mathrm{S} / \mathrm{N}$ values varying between 30 and 680 , and belong to 1388 stars that non-uniformly sample the following intervals of atmospheric parameters: $3442 \mathrm{~K} \leqslant$ $T_{\text {eff }} \leqslant 47250 \mathrm{~K},-2.94 \leqslant[\mathrm{Fe} / \mathrm{H}] \leqslant+1.40$, and $0.00 \leqslant \log g \leqslant$ 4.90 .

The above values are quoted from the source catalog for reference, but our analysis is restricted to only those stars that have literature average atmospheric parameters within the limits defined by our calibration sample $\left(4800 \mathrm{~K} \lesssim T_{\text {eff }} \lesssim 6500 \mathrm{~K}\right.$, $-0.90 \lesssim[\mathrm{Fe} / \mathrm{H}] \lesssim+0.50$, and $3.60 \lesssim \log g \lesssim 4.70)$. We have also restricted our selection to stars with classes 4 and 3 attributed to their average parameters, which means that the typical standard deviations around their mean $T_{\text {eff }}$ and $[\mathrm{Fe} / \mathrm{H}]$

\footnotetext{
${ }^{23} \mathrm{http} / / / \mathrm{www}$. obs.u-bordeaux 1.fr/m2a/soubiran/elodie_library.html
}

values are $62 \mathrm{~K}$ and $0.08 \mathrm{dex}$ and $74 \mathrm{~K}$ and $0.10 \mathrm{dex}$, respectively. ${ }^{24}$ No cuts in $\mathrm{S} / \mathrm{N}$ were applied, but stars with indications of variability or close neighbors were removed. The final ELODIE test sample consists of 219 spectra from 138 unique stars. As for the FEROS sample, these spectra were degraded to the MARVELS resolution following the same procedure described in Section 2.1.

The ELODIE test sample was analyzed with the approach presented in Section 4. All spectra were individually normalized and the EWs of the indices were measured in each of them, as if they belonged to different stars. This was done to check the internal consistency of the atmospheric parameter determinations for a given star based on different spectra, which was found to be good. The EWs of the 92 indices for which the calibrations have $R^{2}>0.9$ were considered. Again, a data-specific tuning was necessary and a correction to the EW scale based on the Sun was performed. Average solar ELODIE EWs were

\footnotetext{
${ }^{24}$ For more details, check

http://www.obs.u-bordeaux 1.fr/m2a/soubiran/elo_stel_param.html.
} 

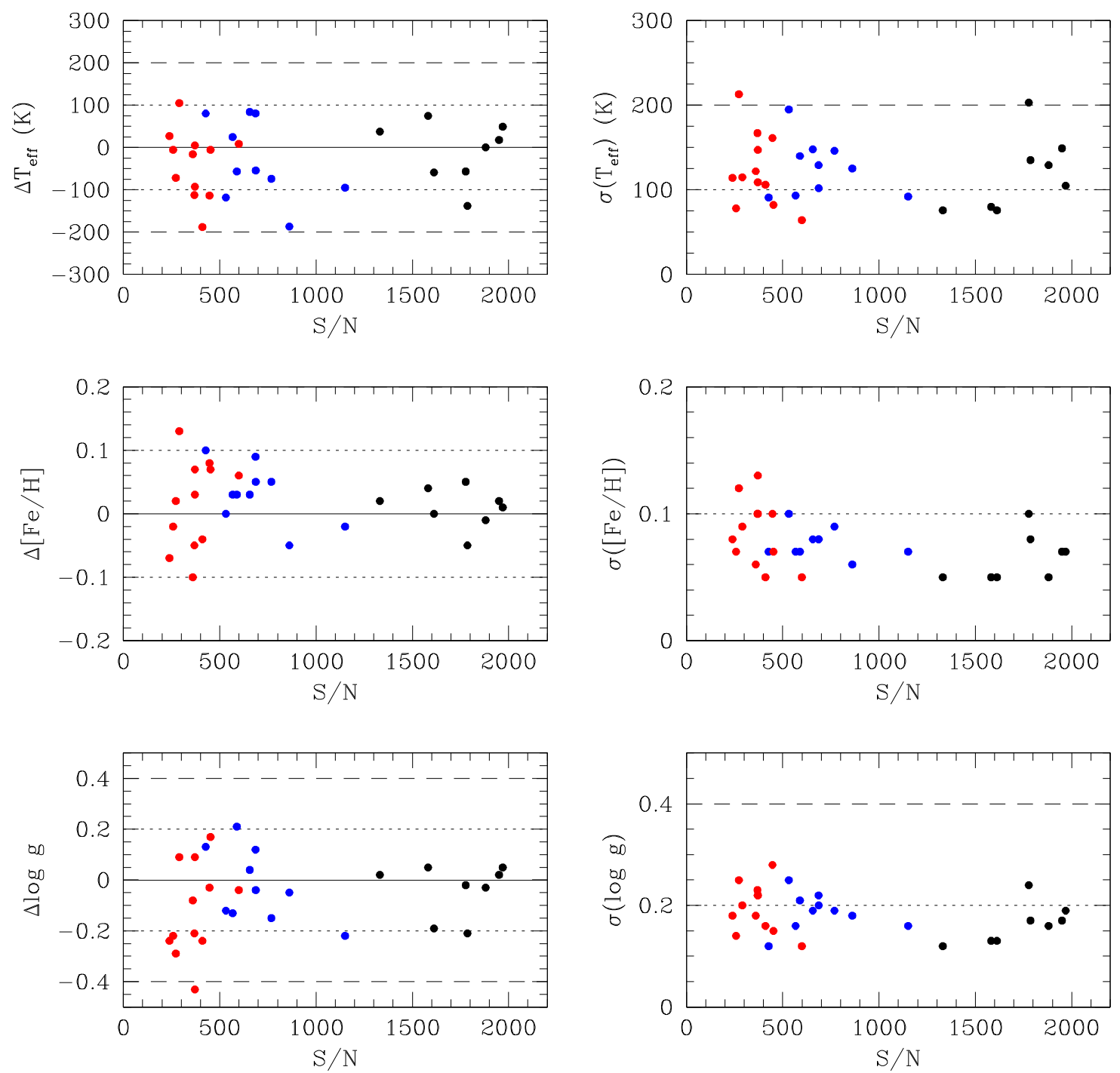

Figure 10. Left column: differences between the atmospheric parameters derived from spectral indices and high-resolution analysis for the MARVELS validation sample as a function of the total S/N. Right column: internal uncertainties of our method as a function of the total S/N. The upper, middle, and lower panels show the cases for $T_{\text {eff }},[\mathrm{Fe} / \mathrm{H}]$, and $\log g$, respectively. Stars with $V<9,9 \leqslant V<11$, and $V \geqslant 11$ are shown by black, blue, and red filled circles, respectively. In all panels, the solid black line represents perfect agreement. The dotted and dashed black lines mark, respectively, the limits \pm 100 and $\pm 200 \mathrm{~K}$ for $T_{\text {eff }}, \pm 0.10$ and \pm 0.20 dex for $[\mathrm{Fe} / \mathrm{H}]$, and \pm 0.20 and \pm 0.40 dex for $\log g$. The differences between the two sets of atmospheric parameters and the internal uncertainties of the spectral indices method do not show any dependencies with $\mathrm{S} / \mathrm{N}$ and $V$ magnitude.

(A color version of this figure is available in the online journal.)

calculated using 11 of the 13 spectra of the Sun with $\mathrm{S} / \mathrm{N}>$ 100. The differences between these average EWs and the values measured on the FEROS solar degraded spectrum were adopted as individual corrections for each index. Using these corrected EWs and the calibrations presented in Section 4.3, atmospheric parameters were derived for the ELODIE sample. The results can be seen in Figure 11 and in Table 5.

We can see that the agreement for $T_{\text {eff }}$ and $[\mathrm{Fe} / \mathrm{H}]$ is good and the average differences and dispersions $(15 \pm 125 \mathrm{~K}$ and $0.06 \pm$ 0.10 , respectively) can be entirely explained by a combination of the internal and external uncertainties from our method with the typical standard deviations around the mean ELODIE parameters. Mild trends are observed for the residuals of these parameters, with the differences between the two sets of results increasing for hot and metal-poor stars, which is expected due to the lower number of calibration stars in these regions of the parameter space. Spectra with low $\mathrm{S} / \mathrm{N}$ values also contribute for the observed trend, since they are clearly associated with the largest residuals (see also Figure 12).
The agreement for $\log g$, on the other hand, is only reasonable, with a relatively large dispersion and clear trends in the residuals. This result was not totally unexpected since the previous tests with the calibration and validation samples have shown that the surface gravity is the most difficult parameter to accurately constrain. Although the average difference and dispersion $(0.07 \pm 0.29)$ can again be explained by a combination of the uncertainties in the two sets of parameters, we can see that some stars have differences higher than 0.5 dex. However, it is clear from the lower panels that these stars are hot and/or metal-poor. Thus, the poor determinations of $T_{\text {eff }}$ and $[\mathrm{Fe} / \mathrm{H}]$ could also be affecting the estimate of $\log g$. Many of these stars also have spectra with low $\mathrm{S} / \mathrm{N}$ values (see Figure 12).

The average solar parameters derived from the 13 spectra available are: $T_{\text {eff }}=5750 \pm 66 \mathrm{~K},[\mathrm{Fe} / \mathrm{H}]=0.00 \pm 0.04$, and $\log g=4.32 \pm 0.11$. The agreement with the canonical solar parameters $\left(T_{\text {eff }}=5777 \mathrm{~K},[\mathrm{Fe} / \mathrm{H}]=0.00\right.$, and $\log g=$ $4.44)$ is very good, with the surface gravity providing the most discrepant result once again. 

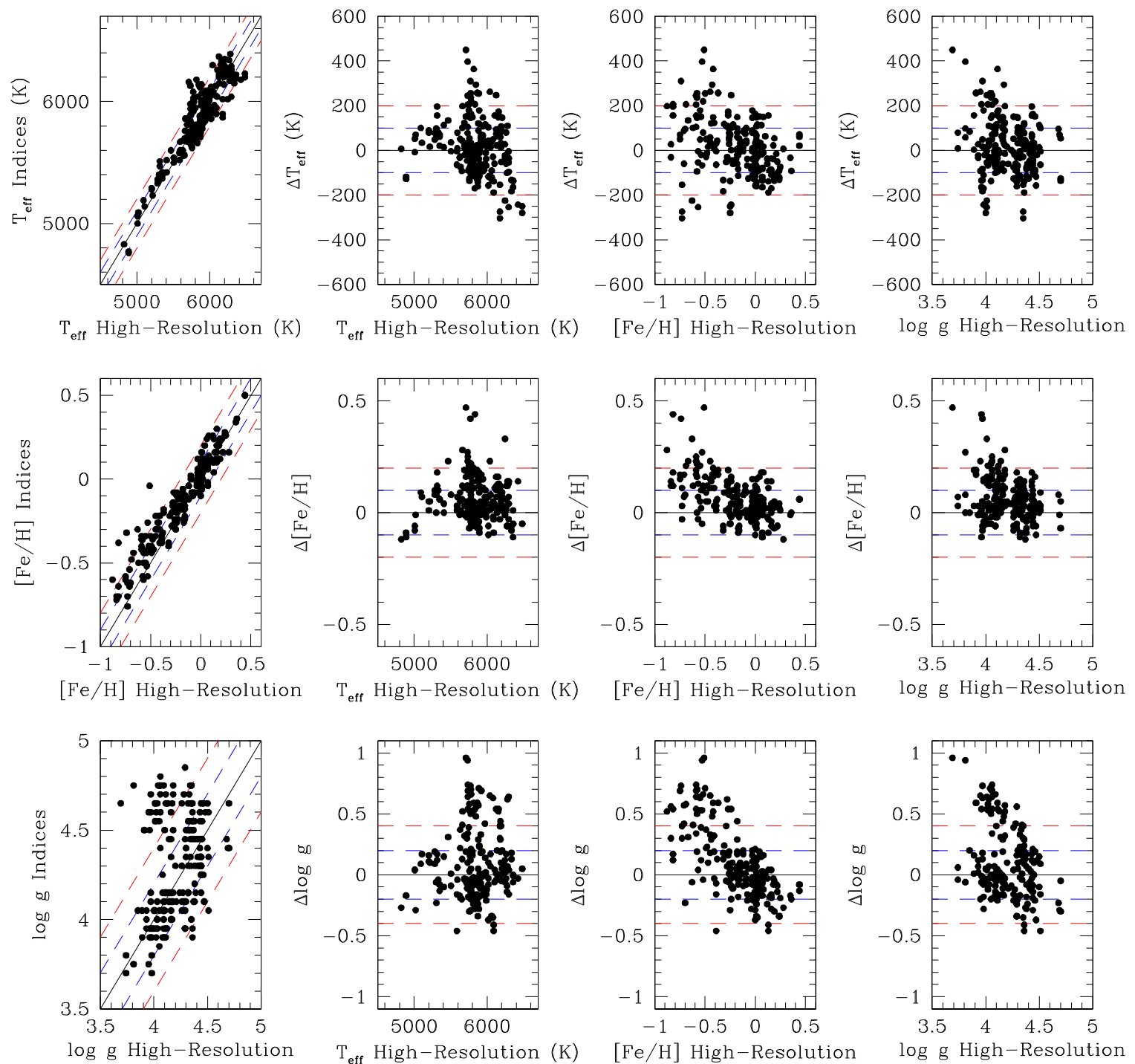

Figure 11. Comparison between atmospheric parameters from spectral indices and the literature for the ELODIE test sample. The panels, symbols, and lines have the same meaning as in Figure 8. Note, however, that the scales are different in some panels. A good agreement between the two sets of atmospheric parameters can be observed as well as small offsets and trends in the residuals.

(A color version of this figure is available in the online journal.)

The ELODIE test sample also offers the opportunity to check the performance of our method for lower $\mathrm{S} / \mathrm{N}$ spectra, which was not possible for the MARVELS validation sample. Figure 12 shows the differences between the spectral indices and ELODIE atmospheric parameters as well as the uncertainties of the former as a function of the $\mathrm{S} / \mathrm{N}$ pixel ${ }^{-1}$ of the spectra. For $\mathrm{S} / \mathrm{N}>200-250$, the typical values of the differences and uncertainties agree well with the ones for the MARVELS sample. At $\mathrm{S} / \mathrm{N} \sim 200-250$, there is a significant increase in both quantities. For $\mathrm{S} / \mathrm{N}<200-250$, the scatter of the distributions stay pretty much constant. These results show that our method is capable of providing reliable results for spectra with lower $\mathrm{S} / \mathrm{N}$, though with lower accuracies than in the high-S/N regime.

The tests discussed above provide an additional validation of the spectral indices method, based on a larger sample of observed stellar spectra with typically lower $\mathrm{S} / \mathrm{N}$ values. As in the previous sections, the precise atmospheric parameters from the literature were recovered with a good precision. The small trends observed in the residuals are caused by the non-uniform sampling of the parameter space by our calibration sample and the lower sensitivities of the indices to the surface gravity.

\section{CONCLUSIONS}

In this paper, we described the usage of spectral indices as a fast and homogeneous approach to determine accurate atmospheric parameters $\left(T_{\text {eff }},[\mathrm{Fe} / \mathrm{H}], \log g\right)$ for samples of FGK dwarfs and subgiants with intermediate $(R \sim 12,000)$ resolution spectra, applied to observations from the MARVELS survey. EWs of the indices were measured on normalized spectra and then compared to values calculated with a set of calibrations, which were constructed using 309 stars with precise atmospheric parameters resulting from the analysis of FEROS spectra. The best solutions were obtained through a $\chi_{r}^{2}$ minimization of the differences between these two sets of EWs. The entire analysis was automatized with three codes developed for this work.

The spectral indices method was validated with a sample of 30 stars, which were observed as part of the MARVELS 

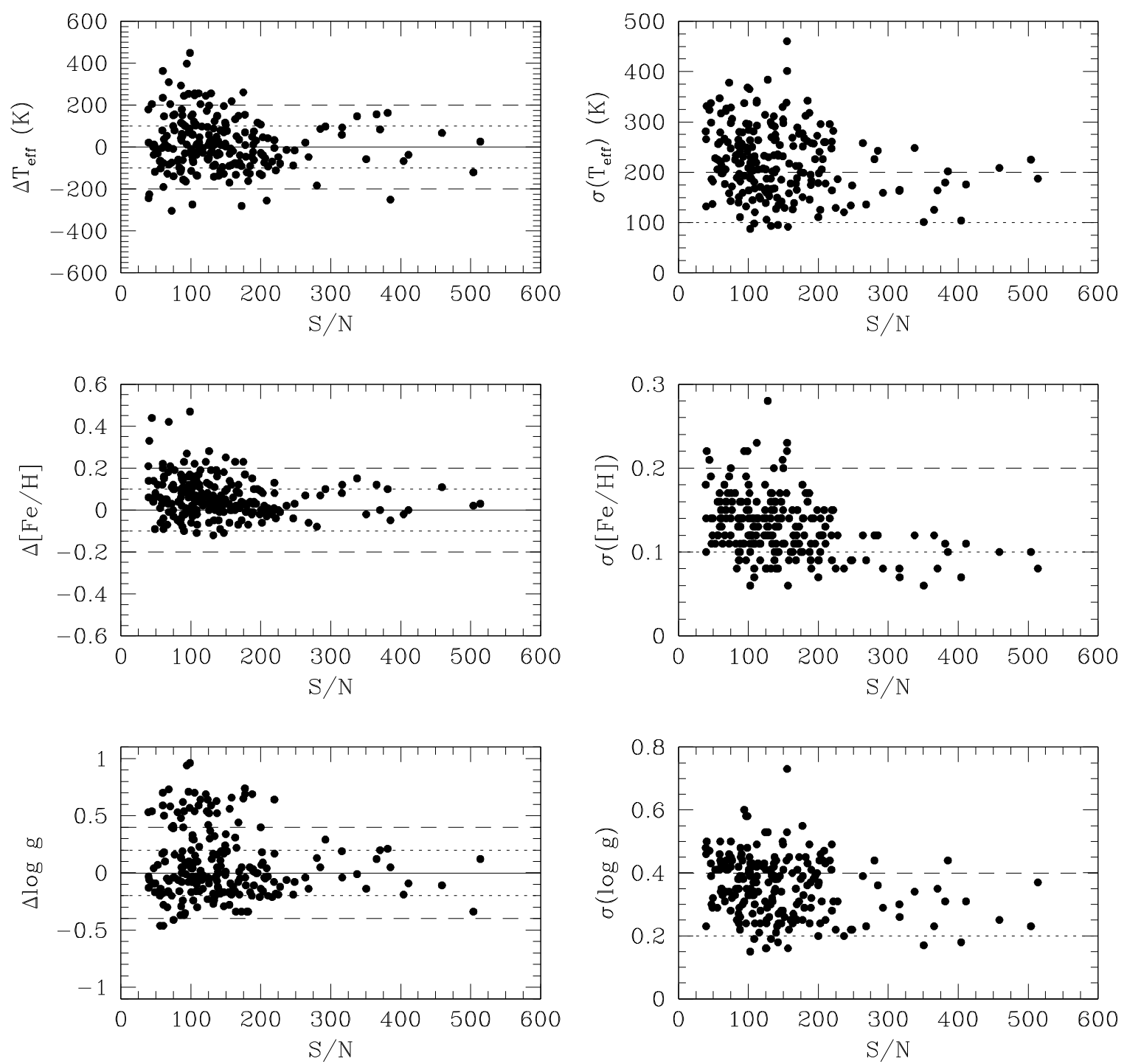

Figure 12. Left column: differences between the atmospheric parameters from spectral indices and from the literature for the ELODIE test sample as a function of $\mathrm{S} / \mathrm{N} \mathrm{pixel}^{-1}$. Right column: internal uncertainties of our method as a function of S/N pixel ${ }^{-1}$. The panels, symbols, and lines have the same meaning as in Figure 10 . Note, however, that the scales are different and no distinctions based on $V$ magnitude are made. The differences between the two sets of atmospheric parameters and the internal uncertainties of the spectral indices method significantly increase for $\mathrm{S} / \mathrm{N}<200-250$.

survey and have precise atmospheric parameters derived from classical model atmosphere analyses. We were able to recover these parameters within $80 \mathrm{~K}$ for $T_{\text {eff }}, 0.05$ dex for $[\mathrm{Fe} / \mathrm{H}]$, and 0.15 dex for $\log g$. The average internal errors of the method are $101 \mathrm{~K}, 0.06 \mathrm{dex}$, and $0.15 \mathrm{dex}$, respectively. These values are consistent with the typical external uncertainties found between different high-resolution spectroscopic analyses (see, e.g., Table 5 of Ghezzi et al. 2010b).

Further confirmation of the good performance of the method was provided by the analysis of a subsample of 138 stars from the ELODIE stellar library with average literature atmospheric parameters with good quality. These were recovered by our method with precisions of $125 \mathrm{~K}$ for $T_{\text {eff }}, 0.10$ dex for $[\mathrm{Fe} / \mathrm{H}]$, and $0.29 \mathrm{dex}$ for $\log g$. The higher uncertainties are caused by a combination of the internal uncertainties of the spectral indices approach, the typically lower $\mathrm{S} / \mathrm{N}$ values for the ELODIE spectra and the typical standard deviations around the mean ELODIE parameters. Undersampled regions in the parameter space for the calibration sample might also play a role. However, these errors are still similar or lower than typical uncertainties provided by the pipelines of other intermediate-resolution surveys (e.g., RAVE; Kordopatis et al. 2013).

This agreement demonstrates that the spectral indices approach is a powerful tool to derive accurate and precise atmospheric parameters for solar-type stars. The method is quite general and its application to a particular data set requires only a small tuning of the input parameters (a list of indices and their measured EWs). The approach has an excellent performance, yielding typical stellar parameter uncertainties comparable to or better than those provided by pipelines that utilize spectral synthesis, such as the ones from the SEGUE, RAVE, and LAMOST surveys. We note, however, that they analyze spectra with lower resolutions, $\mathrm{S} / \mathrm{N}$ values, wavelength coverages, or combinations of these properties. Our method could be adopted by similar surveys, with modifications to the list of indices and calibrations as appropriate for differences in the resolution and wavelength coverage.

The next step of our work is the application of the spectral indices method to the entire MARVELS sample ( $~ 3300$ stars), which contains many faint stars $(V \sim 10-12)$ that were never 
previously analyzed. The atmospheric parameters will then be used to derive additional stellar properties, such as mass, radius, distances, and ages. The kinematic analysis, on the other hand, will follow from the precise RVs determined by MARVELS. With accurate chemical and kinematical information for a large and statistically homogeneous sample, MARVELS can provide valuable contributions to many studies, such as the comparison of the statistical properties of stars with and without companions, the search for correlations between the properties of the companions and their stellar hosts, and Galactic chemical and dynamical evolution in the solar neighborhood. These will be the subjects of subsequent papers.

Funding for SDSS-III has been provided by the Alfred P. Sloan Foundation, the Participating Institutions, the National Science Foundation, and the U.S. Department of Energy Office of Science. The SDSS-III Web site is http://www.sdss3.org/.

SDSS-III is managed by the Astrophysical Research Consortium for the Participating Institutions of the SDSS-III Collaboration including the University of Arizona, the Brazilian Participation Group, Brookhaven National Laboratory, Carnegie Mellon University, University of Florida, the French Participation Group, the German Participation Group, Harvard University, the Instituto de Astrofisica de Canarias, the Michigan State/Notre Dame/JINA Participation Group, Johns Hopkins University, Lawrence Berkeley National Laboratory, Max Planck Institute for Astrophysics, Max Planck Institute for Extraterrestrial Physics, New Mexico State University, New York University, Ohio State University, Pennsylvania State University, University of Portsmouth, Princeton University, the Spanish Participation Group, University of Tokyo, University of Utah, Vanderbilt University, University of Virginia, University of Washington, and Yale University.

Funding for the Brazilian Participation Group has been provided by the Ministério de Ciência e Tecnologia (MCT), Fundação Carlos Chagas Filho de Amparo à Pesquisa do Estado do Rio de Janeiro (FAPERJ), Conselho Nacional de Desenvolvimento Científico e Tecnológico (CNPq), and Financiadora de Estudos e Projetos (FINEP).

We thank Eduardo del Peloso for developing the codes used to normalize the spectra and measure the equivalent widths of the indices. We acknowledge Katia Cunha, Verne Smith, and Daniel Eisenstein for helpful suggestions. We thank the anonymous referee for a thorough reading of the manuscript and detailed comments which helped to improve it.

L.G. acknowledges financial support provided by the PAPDRJ CAPES/FAPERJ Fellowship. L.G. thanks K. Cunha and V. Smith for helping with the acquisition of the FEROS spectra. L.D.F. and D.L.O. acknowledge financial support from CAPES and ESO student fellowships. G.F.P.M. acknowledges the financial support by CNPq (476909/2006-6 and 474972/ 2009-7) and FAPERJ (APQ1/26/170.687/2004) grants. B.X.S. acknowledges support from CNPq (301462/2009-7).

Facilities: Max Planck:2.2m, Sloan, ARC

\section{REFERENCES}

Albrecht, S., Winn, J. N., Johnson, J. A., et al. 2011, ApJ, 738, 50 Ammler-von Eiff, M., Santos, N. C., Sousa, S. G., et al. 2009, A\&A, 507, 523

Bakos, G. Á., Noyes, R. W., Kovács, G., et al. 2007, ApJ, 656, 552

Barbieri, M., Alonso, R., Desidera, S., et al. 2009, A\&A, 503, 601

Bensby, T., \& Feltzing, S. 2006, MNRAS, 367, 1181
Brugamyer, E., Dodson-Robinson, S. E., Cochran, W. D., \& Sneden, C. 2011, ApJ, 738, 97

Burke, C. J., McCullough, P. R., Valenti, J. A., et al. 2007, ApJ, 671, 2115

Casagrande, L., Schönrich, R., Asplund, M., et al. 2011, A\&A, 530, A138

da Silva, R., Udry, S., Bouchy, F., et al. 2007, A\&A, 473, 323

de Laverny, P., Recio-Blanco, A., Worley, C. C., \& Plez, B. 2012, A\&A, 544, A126

De Lee, N., Ge, J., Crepp, J. R., et al. 2013, AJ, 145, 155

De Silva, G. M., Freeman, K. C., Bland-Hawthorn, J., Asplund, M., \& Bessell, M. S. 2007, AJ, 133, 694

del Peloso, E. F., da Silva, L., \& Porto de Mello, G. F. 2005a, A\&A, 434, 275

del Peloso, E. F., da Silva, L., Porto de Mello, G. F., \& Arany-Prado, L. I. 2005b, A\&A, 440, 1153

Edvardsson, B., Andersen, J., Gustafsson, B., et al. 1993, A\&AS, 102, 603

Eisenstein, D. J., Weinberg, D. H., Agol, E., et al. 2011, AJ, 142, 72

Fischer, D. A., Vogt, S. S., Marcy, G. W., et al. 2007, ApJ, 669, 1336

Fleming, S. W., Ge, J., Barnes, R., et al. 2012, AJ, 144, 72

Ge, J., \& Eisenstein, D. 2009, in Astro2010: The Astronomy and Astrophysics Decadal Survey, Science White Papers, 86

Ge, J., Lee, B., de Lee, N., et al. 2009, Proc. SPIE, 7440, 74400L

Ge, J., Mahadevan, S., Lee, B., et al. 2008, in ASP Conf. Ser. 398, Extreme Solar Systems, ed. D. Fischer, F. A. Rasio, S. E. Thorsett, \& A. Wolszczan (San Francisco, CA: ASP), 449

Ghezzi, L., Cunha, K., Schuler, S., \& Smith, V. V. 2010a, ApJ, 725, 721

Ghezzi, L., Cunha, K., Smith, V. V., et al. 2010b, ApJ, 720, 1290

Gonzalez, G., Carlson, M. K., \& Tobin, R. W. 2010, MNRAS, 403, 1368

González Hernández, J. I., Israelian, G., Santos, N. C., et al. 2010, ApJ, 720,1592

Gratton, R. G., Carretta, E., Desidera, S., et al. 2003, A\&A, 406, 131

Gunn, J. E., Siegmund, W. A., Mannery, E. J., et al. 2006, AJ, 131, 2332

Haywood, M. 2008, MNRAS, 388, 1175

Heiter, U., \& Luck, R. E. 2003, AJ, 126, 2015

Jiang, P., Ge, J., Cargile, P., et al. 2013, AJ, 146, 65

Kang, W., Lee, S.-G., \& Kim, K.-M. 2011, ApJ, 736, 87

Karatas, Y., \& Klement, R. J. 2012, NewA, 17, 22

Kaufer, A., Stahl, O., Tubbesing, S., et al. 1999, Msngr, 95, 8

Kordopatis, G., Gilmore, G., Steinmetz, M., et al. 2013, AJ, 146, 134

Lasker, B. M., Lattanzi, M. G., McLean, B. J., et al. 2008, AJ, 136, 735

Laws, C., Gonzalez, G., Walker, K. M., et al. 2003, AJ, 125, 2664

Lee, B. L., Ge, J., Fleming, S. W., et al. 2011, ApJ, 728, 32

Lee, Y. S., Beers, T. C., Sivarani, T., et al. 2008, AJ, 136, 2022

Luck, R. E., \& Heiter, U. 2006, AJ, 131, 3069

Ma, B., Ge, J., Barnes, R., et al. 2013, AJ, 145, 20

Mack, C. E., III, Ge, J., Deshpande, R., et al. 2013, AJ, 145, 139

Mashonkina, L., \& Gehren, T. 2000, A\&A, 364, 249

Mishenina, T. V., Soubiran, C., Bienaymé, O., et al. 2008, A\&A, 489, 923

Moore, C. E., Minnaert, M. G. J., \& Houtgast, J. 1966, The Solar Spectrum $2935 \AA$ to $8770 \AA$ (Washington, DC: National Bureau of Standards Monograph, US Government Printing Office)

Naef, D., Latham, D. W., Mayor, M., et al. 2001, A\&A, 375, L27

Ogando, R. L. C., Maia, M. A. G., Pellegrini, P. S., \& da Costa, L. N. 2008, AJ, 135,2424

Prugniel, Ph., \& Soubiran, C. 2001, A\&A, 369, 1048

Prugniel, Ph., Soubiran, C., Koleva, M., \& Le Borgne, D. 2007, arXiv:astro-ph/0703658v2

Ribas, I., Porto de Mello, G. F., Ferreira, L. D., et al. 2010, ApJ, 714, 384

Robinson, S. E., Ammons, S. M., Kretke, K. A., et al. 2007, ApJS, 169, 430

Robinson, S. E., Strader, J., Ammons, S. M., Laughlin, G., \& Fischer, D. 2006, ApJ, 637, 1102

Saffe, C. 2011, RMxAA, 47, 3

Sánchez-Blázquez, P., Forbes, D. A., Strader, J., Brodie, J., \& Proctor, R. 2007, MNRAS, 377, 759

Santos, N. C., Israelian, G., \& Mayor, M. 2004, A\&A, 415, 1153

Santos, N. C., Israelian, G., Mayor, M., Rebolo, R., \& Udry, S. 2003, A\&A, 398, 363

Siebert, A., Williams, M. E. K., Siviero, A., et al. 2011, AJ, 141, 187

Smolinski, J. P., Lee, Y. S., Beers, T. C., et al. 2011, AJ, 141, 89

Sozzetti, A., Torres, G., Charbonneau, D., et al. 2007, ApJ, 664, 1190

Steinmetz, M., Zwitter, T., Siebert, A., et al. 2006, AJ, 132, 1645

Stempels, H. C., Collier Cameron, A., Hebb, L., Smalley, B., \& Frandsen, S. 2007, MNRAS, 379, 773

Torres, G., Bakos, G. Á., Kovács, G., et al. 2007, ApJL, 666, L121

Torres, G., Fischer, D. A., Sozzetti, A., et al. 2012, ApJ, 757, 161

Trager, S. C., Faber, S. M., Worthey, G., \& González, J. J. 2000, AJ, 120, 165

Valenti, J. A., \& Fischer, D. A. 2005, ApJS, 159, 141

Wang, J., Ge, J., Wan, X., Lee, B., \& De Lee, N. 2012a, PASP, 124, 598

Wang, J., Ge, J., Wan, X., Lee, B., \& De Lee, N. 2012b, PASP, 124, 1159 
Wang, S., Hildebrand, R. H., Hobbs, L. M., et al. 2003, Proc. SPIE, 4841, 1145 Wisniewski, J. P., Ge, J., Crepp, J. R., et al. 2012, AJ, 143, 107

Worley, C. C., de Laverny, P., Recio-Blanco, A., et al. 2012, A\&A, 542, A48

Worthey, G., Faber, S. M., Jesús González, J., \& Burstein, D. 1994, ApJS, 94, 687

Wright, J. T., Fischer, D. A., Ford, E. B., et al. 2009, ApJL, 699, L97

Wright, J. T., Roy, A., Mahadevan, S., et al. 2013, ApJ, 770, 119

Wu, Y., Luo, A.-L., Li, H.-N., et al. 2011, RAA, 11, 924
Yanny, B., Rockosi, C., Newberg, H. J., et al. 2009, AJ, 137, 4377

York, D. G., Adelman, J., Anderson, J. E., Jr., et al. 2000, AJ, 120, 1579

Zieliński, P., Niedzielski, A., Wolszczan, A., Adamów, M., \& Nowak, G. 2012, A\&A, 547, A91

Zucker, D. B., de Silva, G., Freeman, K., Bland-Hawthorn, J., \& Hermes Team 2012, in ASP Conf. Ser. 458, Galactic Archaeology: Near-Field Cosmology and the Formation of the Milky Way, ed. W. Aoki, M. Ishigaki, T. Suda, T. Tsujimoto, \& N. Arimoto (San Francisco, CA: ASP), 421 